\title{
The Cosmic Energy Inventory
}

\author{
Masataka Fukugita \\ Institute for Advanced Study, Princeton, NJ 08540 USA \\ and \\ Institute for Cosmic Ray Research, University of Tokyo, \\ Kashiwa 277-8582, Japan \\ P. J. E. Peebles \\ Joseph Henry Laboratories, Princeton University, \\ Princeton, NJ 08544 USA
}

\begin{abstract}
We present an inventory of the cosmic mean densities of energy associated with all the known states of matter and radiation at the present epoch. The observational and theoretical bases for the inventory have become rich enough to allow estimates with observational support for the densities of energy in some 40 forms. The result is a global portrait of the effects of the physical processes of cosmic evolution.
\end{abstract}

Subject headings: cosmology

\section{Introduction}

There is now a substantial observational basis for estimates of the cosmic mean densities of all the known and more significant forms of matter and energy in the present-day universe. The compilation of the energy inventory offers an overview of the integrated effects of the energy transfers involved in all the physical processes of cosmic evolution operating on scales ranging from the Hubble length to black holes and atomic nuclei. The compilation also offers a way to assess how well we understand the physics of cosmic evolution, by the degree of consistency among related entries. Very significant observational advances, particularly from large-scale surveys including the Two Degree Field Galaxy Redshift Survey (2dF: Colless et al. 2001), the Sloan Digital Sky Survey (SDSS: York et al. 2000; Abazajian et al. 2003), the Two Micron All-Sky Survey (2MASS: Huchra et al. 2003), the HI Parkes All Sky Survey (HIPASS: Zwaan et al. 2003), and the Wilkinson Microwave Anisotropy Probe (WMAP: Bennett et al. 2003a), make it timely to compile what is known about the entire energy inventory.

We present here our choices for the categories and estimates of the entries in the inventory. Many of the arguments in this exercise are updated versions of what is in the literature. Some arguments are new, as is the adoption of a single universal unit - the density parameter - that makes comparisons across a broad variety of forms of energy immediate, but the central new development is that the considerable range of consistency checks demonstrates that many of the entries in the inventory are meaningful and believable.

People have been making inventories for a long time. The medieval Domesday Book (1086-7) gave King William a picture of the wealth and organization of the kingdom (and it gives us fascinating insight into a society). The present-day cosmic energy inventory similarly gives us a picture of the amount and organization of the material contents of the universe. It also offers us a way to assess the reliability of our picture, through checks of consistency. A early example of the latter point is de Sitter's (1917) discussion of Ein- 
stein's (1917) static homogeneous universe. Under the assumption that observations can reach a fair sample of the universe, one can seek constraints on the cosmic mean mass density and space curvature and test the predicted relation between the two. Hubble's (1929) redshift-distance relation led to a revision of the predicted relation between the mass density and space curvature, and his use of redshifts to convert galaxy counts into number densities greatly improved the estimate of the mean mass density (Hubble 1934). ${ }^{1}$ One may also consider the relations among the mean luminosity density of the galaxies, the production of the heavy elements, and the surface brightness of the night sky (Partridge \& Peebles 1967; Peebles \& Partridge 1967); the relation between galaxy colors, the initial mass function, and the star formation history (Searle, Sargent, \& Bagnuolo 1973); the relation between the last two sets of considerations (Tinsley 1973); the relation between the light element abundances and the baryon mass density (Gott et al. 1974); and the relation between the luminosity density of the quasars and the mean mass density in quasars and their remnants (Soltan 1982). Basu and Lynden-Bell (1990) show how one can analyze what is learned from this rich set of considerations in terms of an entropy inventory. We have chosen instead to base this discussion on an energy inventory.

Our inventory includes the mass densities in the various states of baryons. This is an updated version of the baryon budget of Fukugita, Hogan \& Peebles (1998; FHP). Most entries in this part of the inventory have not changed much in the past half decade, while substantial advances in the observational constraints have considerably reduced the uncertainties. It appears that most of the baryonic components are observationally well constrained, apart from the largest entry, for warm plasma, which still is driven by the need to balance the budget rather than more directly by the observations.

The largest entries, for dark matter and the cosmological constant, or dark energy, are well constrained within a cosmological theory that is rea-

\footnotetext{
${ }^{1}$ Hubble's (1936) estimate based on galaxy masses derived from the velocity dispersion in the Virgo Cluster, which takes account of what is now termed nonbaryonic dark matter, translates to density parameter $\Omega_{m} \sim 0.1$, impressively close to the modern value.
}

sonably well tested, but the physical natures of these entries remain quite hypothetical. We understand the physical natures of magnetic fields and cosmic rays, but the theories of the evolution of these components, and the estimates of their contributions to the present energy inventory, are quite uncertain. The situation for most of the other entries tends to be between these extremes: the physical natures of the entries are adequately characterized, for the most part, and our estimates of their energy densities, while generally not very precise, seem to be meaningfully constrained by the observations.

Several cautionary remarks are in order. First, some types of energy are not readily expressed as sums of simple components; we must adopt conventions. Second, there is no arrangement of categories that offers a uniquely natural place for each component; we must again adopt conventions. Perhaps further advances in the understanding of cosmic evolution will lead to a more logically ordered inventory. Third, it is arguably artificial to represent binding energies as very small negative density parameters. The advantage is that it simplifies comparisons across the entire inventory. Fourth, the amount of space we devote is in accord with the importance of the issues of physics and astronomy, not with the value of the energy. For instance, the major components in the inventory are dark energy and dark matter, but they are physically simple in that their work is only gravitational, so our discussion is rather short. Dissipative energies are small in absolute values, but their physical significance is large, and our discussions are on occasion rather lengthy. Finally, it is a task for future work to make some of our estimates more accurate by using data and computations that exist but are difficult to assemble. We mention the main examples in $\S 3$.

The inventory, which is presented in Table 1, is arranged by categories and components within categories. The explanations of conventions and sources for each entry are presented in $\S 2$, in subsections with numbers keyed to the category numbers in the first column of the table. Our discussion of checks of the entries is not so simply ordered, because the checks depend on relations among considerations of entries scattered through the table. A guide to the considerable variety of checks detailed in $\S 2$ is presented in $\S 3$. 
TABLE 1

The Cosmic Energy Inventory

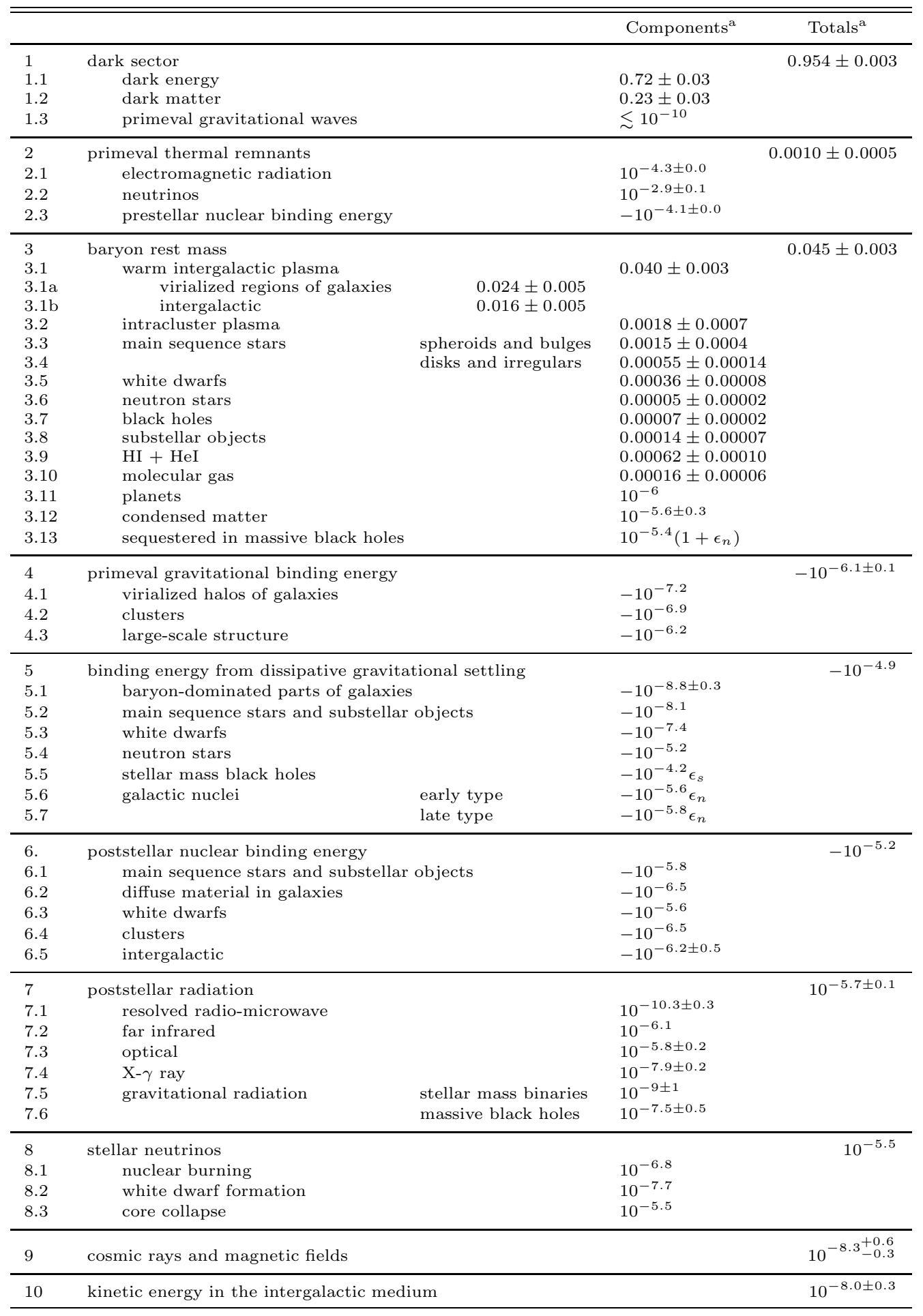

${ }^{\mathrm{a}}$ Based on Hubble parameter $h=0.7$. 


\section{The Energy Inventory}

The inventory in Table 1 assumes the now standard relativistic Friedmann-Lemaître $\Lambda$ CDM cosmology, in which space sections at fixed world time have negligibly small mean curvature, ${ }^{2}$ Einstein's cosmological term, $\Lambda$, is independent of time and position, the dark matter is an initially cold noninteracting gas, and primeval departures from homogeneity are adiabatic, Gaussian, and scale-invariant. Physics in the dark sector is not well constrained: $\Lambda$ might be replaced with a dynamical component, ${ }^{3}$ as in the models for dark energy now under discussion, the physics of the dark matter may prove to be more complicated than that of a free collisionless gas, and the initial conditions may not be adequately approximated by the present standard cosmology. If such complications were present we expect their effects on entries that are sensitive to the cosmological model would be slight, however, because the cosmological tests now offer close to compelling evidence that the $\Lambda \mathrm{CDM}$ model is a useful approximation to reality (Bennett et al. 2003a; Spergel et al. 2003; Tegmark et al. 2004a; and references therein).

To help simplify the discussion we adopt a nominal distance scale, corresponding to Hubble's constant $^{4}$

$$
H_{o}=70 \mathrm{~km} \mathrm{~s}^{-1} \mathrm{Mpc}^{-1} \text {. }
$$

The energy density, $\bar{\rho}_{i}$, in the form of component $i$ is expressed as a density parameter,

$$
\Omega_{i}=\frac{8 \pi G \bar{\rho}_{i}}{3 H_{o}^{2}} .
$$

Since $H_{o}$ is at best measured to ten percent accuracy (Freedman et al. 2001), an improved distance scale could produce noticeable revisions to the inventory.

\footnotetext{
${ }^{2}$ The most compelling evidence is from the position of the first peak in the anisotropy power spectrum of the of cosmic microwave background temperature field. The WMAP measurements (Bennett et al. 2003a) indicate $\Omega_{m}+\Omega_{\lambda}=$ $1.02 \pm 0.02$. In this paper we simplify the discussion by assuming strictly flat space sections.

${ }^{3}$ The current limit on the index of the equation of state for the dark energy is $w=p / \rho<-0.78$ at 95\% (Bennett $2003 \mathrm{a})$; the bound $w=-1.02_{-0.19}^{+0.13}$ is obtained from the Type Ia supernova Hubble diagram under the assumption of flat space curvature (Riess et al. 2004)

${ }^{4}$ We also write $H_{o}=100 h \mathrm{~km} \mathrm{~s}^{-1} \mathrm{Mpc}^{-1}$, where convenient, but all entries in the inventory in Table 1 assume $h=0.7$.
}

The second column from the right in Table 1 lists the density parameters in the components, and the last column presents the total for each category. Both columns sum to unity. The statement of errors requires some explanation. Most of the errors in categories 1 through 3 are rather well documented. The uncertainties in entries 2.1 and 2.3 are too small to be relevant for the purpose of this inventory. Elsewhere a numerical value stated to one significant figure after the decimal might be supposed to be reliable to roughly $\pm 0.1 \mathrm{dex}$, or about $30 \%$. Where no digit is presented after the decimal point we hope our estimate might within a factor of ten of the real value.

\subsection{The Dark Sector}

The components in category 1 interact with the contents of the visible sector only by gravity, as far as is now known. This makes it difficult to check whether the dark energy - or Einstein's cosmological constant, $\Lambda$ - and the dark matter really have the simple properties assumed in the $\Lambda \mathrm{CDM}$ cosmology. Future versions of the inventory might contain separate entries for the potential, kinetic and gradient contributions to the dark energy density, or a potential energy component in the dark matter.

There is abundant evidence that the total mass density - excluding dark energy - is well below the Einstein-de Sitter value. That means, among other things, that the consistency of crosschecks from the many ways to estimate the mass density provides close to compelling evidence that the gravitational interaction of matter at distances up to the large-scale flows is well approximated by the inverse square law, and that starlight is a good tracer of the mass distribution on scales $\gtrsim 100 \mathrm{kpc}^{5}$

An example that illustrates the situation, and will be used later, is the estimate from weak lensing of the mean galaxy surface mass density contrast,

$$
\Sigma(<y)-\Sigma(y)=A(h y / 1 \mathrm{Mpc})^{-\alpha},
$$

\footnotetext{
${ }^{5}$ For early discussions see Peebles 1986 and Bahcall, Lubin \& Dorman (1995). Recent reviews of the situation are in Fukugita (2001), Peebles \& Ratra (2003), and Bennett et al. (2003a). The present observational situation is reviewed in footnote 6 .
} 
where $\Sigma(y)$ is the ensemble average surface mass density at projected distance $y$ from a galaxy and $\Sigma(<y)$ is the mean surface density within distance $y$. The measurements by McKay et al. (2001) yield $A=2.5_{-0.8}^{+0.7} h m_{\odot} \mathrm{pc}^{-2}$ and $\alpha=-0.8 \pm 0.2$, and they indicate that the power law is a good approximation to the measurements in the range of projected radii

$$
70 \mathrm{kpc} \lesssim y \lesssim 1 \mathrm{Mpc} .
$$

If the galaxy autocorrelation function,

$$
\xi(r)=\left(\frac{r_{o}}{r}\right)^{\gamma}, \gamma=1.77, r_{o}=5 h^{-1} \mathrm{Mpc},
$$

is a good approximation to the galaxy-mass cross correlation function on the range of scales in equation (4), then the mean surface density is

$$
\begin{aligned}
\Sigma(y) & =\rho_{m} r_{o}^{\gamma} y^{1-\gamma} H_{\gamma}, \\
H_{\gamma} & =\frac{\Gamma(1 / 2) \Gamma((\gamma-1) / 2)}{\Gamma(\gamma / 2)} .
\end{aligned}
$$

This agrees with equation (3) if the density parameter belonging to the mean density $\rho_{m}$ of the mass that clusters with the galaxies is

$$
\Omega_{m}(\text { weak lensing })=0.20_{-0.05}^{+0.06} .
$$

This is in the range of estimates of the value of this parameter now under discussion, consistent with the assumption that galaxies are useful tracers of mass.

Our adopted value for the total mass density in nonrelativistic matter, dark plus baryonic, is

$$
\Omega_{m}=\Omega_{\mathrm{DM}}+\Omega_{\mathrm{b}}+\Omega_{\nu}=0.28 \pm 0.03 .
$$

This is in the range of most current estimates. ${ }^{6}$ The measurement may not be tightly constrained,

\footnotetext{
${ }^{6}$ Spergel et al. derived $\Omega_{m} h^{2}=0.13-0.14$ from WMAP either with or without using the constraint from the power spectrum of the $2 \mathrm{dF}$ galaxy distribution. This gives $\Omega_{m}=$ $0.265-0.286$ at our fiducial $h=0.7$. Tegmark et al. (2004a) obtained the central value $\Omega_{m} h^{2}=0.14$ with WMAP data alone, and 0.145 with the constraint from the SDSS galaxy clustering. For $h=0.7$ these values are respectively $\Omega_{m}=0.29$ and 0.30 . The estimate from the $2 \mathrm{dF}$ GRS power spectrum (W. Percival and the 2DFGRS team, 2004 , private communication) is $\Omega_{m} h=0.164 \pm 0.016$, or $\Omega_{m}=0.23 \pm 0.02$ at our distance scale. This is 1.4 standard deviations below equation (8). The lower estimate of $\Omega_{m}$ is due to the somewhat larger value of the small-scale power spectrum compared to SDSS. Note that departures
}

however, and there is the possibility of adjustment of this important parameter beyond the error flag in equation (8).

We make use of the fact that equation (3) is close to the limiting isothermal sphere mass distribution,

$$
\rho(r)=\sigma^{2} / 2 \pi G r^{2}
$$

If we connect this form to the power law in equation (5) at the nominal virial radius $r_{v}$ defined by $\rho\left(<r_{v}\right) / \rho_{c}=200$, we obtain

$$
r_{v}=220 h^{-1} \mathrm{kpc}, \quad \sigma=160 \mathrm{~km} \mathrm{~s}^{-1} .
$$

This measure of the characteristic one-dimensional velocity dispersion, $\sigma$, in luminous galaxies agrees with the mean in quadrature,

$$
\left\langle\sigma^{2}\right\rangle^{1 / 2} \simeq 160 \mathrm{~km} \mathrm{~s}^{-1}
$$

weighted by the FHP morphology fractions, of the dispersions $\sigma=225 \mathrm{~km} \mathrm{~s}^{-1}$ for elliptical galaxies, $\sigma=206 \mathrm{~km} \mathrm{~s}^{-1}$ for S0 galaxies (de Vaucouleurs \& Olson 1982) and $\sigma=136 \mathrm{~km} \mathrm{~s}^{-1}$ for spiral galaxies (Sakai et al. 2000), all at luminosity $L_{B}=L_{B}^{*}$. The isothermal sphere model defines a characteristic mass $M_{v}$ within $r_{v}$. The ratio of $M_{v}$ to the characteristic galaxy luminosity (eq. [(53] below) is

$$
M_{v} / L_{r}=180 h, \quad M_{v} / L_{B}=250 h,
$$

in Solar units. This is consistent with the estimate of $M / L$ within $220 h^{-1} \mathrm{kpc}$ from weak lensing shear (McKay et al. 2001). ${ }^{7}$

The product of $M_{v}$ with the effective number density of luminous $\left(L^{*}\right)$ galaxies, $n_{g}=0.017 h^{3}$ $\mathrm{Mpc}^{-3}$ (eq [52]), gives an estimate that the mean

from the standard assumptions, including flat space curvature, scale-invariant initial conditions, and neglible tensor perturbations, could lead to changes beyond the quoted errors. We also refer to estimates from the Type Ia sypernova redshift-magnitude relation, $\Omega_{m}=0.29_{-0.03}^{+0.05}$ (Riess et al. 2004 ), and from dynamics, including $\Omega_{m}=0.17 \pm 0.05$ from the cluster mass function as a function of redshift (Bahcall $\&$ Bode 2003) and $\Omega_{m}=0.30 \pm 0.08$ from the redshift space two-point correlation function (Hawkins et al. 2003), under the assumption that the bias parameter is $b=1$ (Verde et al. 2002).

${ }^{7}$ It is worth noting that equation (12) is not far from Zwicky's (1933) dynamical estimate for the Coma Cluster, $M / L \sim 100$ at our distance scale. 
mass fraction within the virial radii of the large galaxies,

$$
\frac{\rho\left(<r_{v}\right)}{\rho_{m}}=0.6 .
$$

That is, we estimate that about $60 \%$ of the dark matter is gathered within the virialized parts of normal galaxies.

We consider now how the estimate of $\Omega_{m}$ in equation (8) compares to the estimate from the mass-to-light ratio and the integrated mean luminosity density. The estimates from the SDSS broad-band galaxy luminosity functions are (Yasuda et al. in preparation; see also Blanton et al. 2003)

$$
\begin{aligned}
\mathcal{L}_{B} & =(1.9 \pm 0.2) \times 10^{8} h L_{\odot} \mathrm{Mpc}^{-3} \\
\mathcal{L}_{r} & =(2.3 \pm 0.2) \times 10^{8} h L_{\odot} \mathrm{Mpc}^{3} \\
\mathcal{L}_{z} & =(3.6 \pm 0.4) \times 10^{8} h L_{\odot} \mathrm{Mpc}^{-3} .
\end{aligned}
$$

These densities are the values at $z \approx 0.05$. The value of $\mathcal{L}_{B}$ is inferred from the densities in other color bands. ${ }^{8}$ The luminosity density in the $z$ band is quoted for the later use. The product of the luminosity density with $M / L$ in equation (12) yields the density parameter in matter within the virial radii of galaxies, $\Omega_{m, v}=0.18$ and 0.16 for the $B$ and $r$ bands, respectively. On dividing by equation (13) we arrive at $\Omega_{m}=0.31$ and 0.27 , consistent with equation (8).

Note that $M / L$ in equation (12) is about half the value estimated for rich clusters, $(M / L)_{\text {cluster }}=$ $450 \pm 100$ (FHP). In rich clusters all mass in the outskirts of galaxies, beyond their virial radii, is integrated in the mass estimate, so that it is reasonable to suppose that it gives a larger value by the inverse factor in equation (13). When the cluster value for $M / L$ is multiplied by the $B$ band luminosity density we get $\Omega_{m}=0.32$, consistent with the other estimates.

Entry 1.3 assumes inflation has produced gravitational waves with a scale-invariant spectrum, meaning the strain $\delta$ appearing at the Hubble length is independent of epoch. The density parameter associated with gravitational waves with wavelengths on the order of the Hubble length is

${ }^{8}$ The ratios of luminosity densities, $1: 1.20: 1.87$, are approximately what is expected from the average colors, $\langle B-r\rangle=1.0$ and $\langle r-z\rangle=0.6\left[(B-r)_{\odot}=0.82\right.$, $(r-z) \odot=0.12]$.
$\Omega_{g} \sim \delta^{2}$, and the absence of an appreciable effect of gravitational waves on the anisotropy of the $3 \mathrm{~K}$ thermal cosmic background radiation indicates $\delta \lesssim 10^{-5}$. The gravitational waves produced by cosmic phase transitions, if detected or convincingly predicted, might be entered in this category. Gravitational waves from the relativistic collapse of stars and galactic nuclei are included in category 7.

The other entries in the first category in Table 1 are computed from equation (8) and our estimates of the other significant contributions to the total mass density, under the assumption that the density parameters sum to unity, that is, space curvature is neglected.

\subsection{Thermal Remnants}

\subsubsection{Cosmic Background Radiation}

Entry 2.1 is based on the COBE measurement of the temperature of the thermal cosmic electromagnetic background radiation (the CMBR), $T_{o}=2.725 \mathrm{~K}$ (Mather et al. 1999). The COBE and UBC measurements (Mather et al. 1990; Gush, Halpern, \& Wishnow 1990) show that the spectrum is very close to thermal. It has been slightly disturbed by the observed interaction with the hot plasma in clusters of galaxies (LaRoque et al. 2003 and references therein). The limit on the resulting fractional increase in the radiation energy density is (Fixsen et al. 1996)

$$
\delta u / u=4 y<6 \times 10^{-5} .
$$

This means that the background radiation energy density has been perturbed by the amount $\Delta \Omega<10^{-8.5}$. Improvements of this number are under discussion (e.g. Zhang, Pen \& Trac 2004), and might be entered in a future version of the inventory.

The thermal background radiation has been perturbed also by the dissipation of the primeval fluctuations in the distributions of baryons and radiation on scales smaller than the Hubble length at the epoch of decoupling of baryonic matter and radiation. If the initial mass fluctuations are adiabatic and scale-invariant the fractional perturbation to the radiation energy per logarithmic increment of the comoving length scale is $\delta u / u \sim \delta_{h}^{2}$, where $\delta_{h} \sim 10^{-5}$ is the density contrast appearing at the Hubble length. This is small compared 
to the subsequent perturbation by hot plasma (eq [15]).

Entry 2.2 uses the standard estimates of the relict thermal neutrino temperature, $T_{\nu}=$ $(4 / 11)^{1 / 3} T_{o}$, and the number density per family, $n_{\nu}=112 \mathrm{~cm}^{-3}$. We adopt the neutrino mass differences from oscillation experiments (Fukuda et al. 1998; Kameda et al. 2001; Eguchi, et al. 2003; Bahcall \& Peña-Garay 2003),

$$
\begin{aligned}
& m_{\nu_{3}}^{2}-m_{\nu_{2}}^{2}=0.002 \pm 0.001 \mathrm{eV}^{2} \\
& m_{\nu_{2}}^{2}-m_{\nu_{1}}^{2}=6.9 \times 10^{-5} \mathrm{eV}^{2}
\end{aligned}
$$

where the neutrino mass eigenstates are ordered as $m_{\nu_{1}}<m_{\nu_{2}}<m_{\nu_{3}}$. Entry 2.2 , the density parameter $\Omega_{\nu}$ in primeval neutrinos, assumes that $m_{\nu_{e}}$ may be neglected. The upper limit from WMAP and SDSS is $\Omega_{\nu}<0.04$ (Tegmark et al. 2004a). At this limit the three families would have almost equal masses, $m_{\nu}=0.6 \mathrm{eV}$. This may not be very likely, but one certainly must bear in mind the possibility that our entry is a considerable underestimate.

\subsubsection{Primordial Nucleosynthesis}

Light elements are produced as the universe expanded and cooled through $k T \sim 0.1 \mathrm{MeV}$, in amounts that depend on the baryon abundance. The general agreement of the baryon abundance inferred in this way with that derived from the CMBR temperature anisotropy gives confidence that the total amount of baryons - excluding what might have been trapped in the dark matter prior to light element nucleosynthesis - is securely constrained.

Estimates of the baryon density parameter from the WMAP and SDSS data (Spergel et al. 2003; Tegmark et al. 2004a), and from the deuterium (Kirkman et al. 2003) and helium abundance measurements (Izotov \& Thuan 2004) are, respectively, $\Omega_{\mathrm{b}} h^{2}=0.023 \pm 0.001,0.0214 \pm 0.0020$, and $0.013_{-0.001}^{+.002}$, where the last number is the allsample average for helium from Izotov \& Thuan. We adopt

$$
\Omega_{\mathrm{b}} h^{2}=0.0225 \pm 0.0015,
$$

close to the mean of the first two. Since the relation between the helium abundance and the baryon density parameter has a very shallow slope, an accurate abundance estimate (say, with $<1 \%$ error) is needed for a strong constraint on $\Omega_{\mathrm{b}} h^{2}$. We consider that the current estimates may still suffer from systematic errors which are not included in the error estimates in the literature. ${ }^{9}$ Within the standard cosmology our adopted value in equation (17) requires that the primeval helium abundance is

$$
Y_{p}=0.248 \pm 0.001
$$

and the ratio of the total matter density to the baryon component is

$$
\Omega_{m} / \Omega_{b}=6.11 \pm 0.89
$$

We need in later sections the stellar helium production rate with respect to that of the heavy elements. The all-sample analysis of Izotov \& Thuan (2004) gives $\Delta Y / \Delta Z \simeq 2.8 \pm 0.5$. The value derived by Peimbert, Peimbert \& Ruiz (2000) corresponds to $2.3 \pm 0.6$. These value may be compared to estimates from the perturbative effects on the effective temperature-luminosity relation for the atmosphere of main sequence dwarfs, $\Delta Y / \Delta Z \simeq 3 \pm 2$ (Pagel \& Portinari 1998), and $2.1 \pm 0.4$ (Jimenez et al. 2003). From the initial elemental abundance estimate in the standard solar model of Bahcall, Pinsonneault \& Basu (2001; hereinafter BP2000) we derive $\Delta Y / \Delta Z \simeq 1.4$. We adopt

$$
\Delta Y / \Delta Z \simeq 2 \pm 1
$$

Nuclear binding energy was released during nucleosynthesis. This appears in entry 2.3 as a negative value, meaning the comoving baryon mass density has been reduced and the energy density in radiation and neutrinos increased. The effect on the radiation background has long since been thermalized, of course, but the entry is worth recording

\footnotetext{
${ }^{9}$ We note, as an indication of the difficulty of these observations, that helium abundances inferred from the triplet $4 d-2 p$ transition $(\lambda 4471)$ are lower than what is indicated by the triplet $3 \mathrm{~d}-2 \mathrm{p}(\lambda 5876)$ and singlet $3 \mathrm{~d}-2 \mathrm{p}(\lambda 6678)$ transitions, by an amount that is significantly larger than the quoted errors. Another uncertainty arises from stellar absorption corrections, which are calculated only for the $\lambda 4471$ line. The table given in Izotov and Thuan suggests that a small change in absorption corrections for the $\lambda 4471$ line induces a sizable change in the final helium abundance estimate. We must remember also that the value of $\Delta Y / \Delta Z$, which is needed to derive $Y_{p}$, is not very well determined.
} 
for comparison to the nuclear binding energy released in stellar evolution. For the same reason, we compute the binding energy relative to free protons and electrons. The convention is artificial, because light element formation at high redshifts was dominated by radiative exchanges of neutrons, protons and atomic nuclei, and the abundance of the neutrons was determined by energy exchanges with the cosmic neutrino background. It facilitates comparison with category 6 , however. The nuclear binding energy in entry 2.3 is the product

$$
-\Omega_{\mathrm{NB}, \mathrm{He}}=0.0071 Y_{p} \Omega_{\mathrm{b}}=10^{-4.1} .
$$

This is larger in magnitude than the energy in the CMBR today.

\subsection{The Baryon Rest Mass Budget}

The entries in this category refer to the baryon rest mass: one must add the negative binding energies to get the present mass density in baryons. The binding energies are small and the distinction purely formal at the accuracy we can hope for in cosmology, of course, with the conceivable exception of the baryons sequestered in black holes.

We begin with the best-characterized components, the stars, star remnants, and planets. We then consider the diffuse components, and conclude this subsection with discussions of the baryons in groups and the intergalactic medium and the lost baryons in black holes.

\subsubsection{Stars}

This is an update of the analysis in FHP. Following the same methods, we estimate the baryon mass in stars from the galaxy luminosity density and the stellar mass-to-light ratio, $M_{\text {stars }} / L$, along with a stellar initial mass function (the IMF) that allows us to estimate the mass fractions in various forms of stars and star remnants.

Kauffmann et al. (2003) present an extensive analysis of the stellar mass-to-light ratio based on ugriz photometry for $10^{5}$ SDSS galaxies and a population synthesis model (Bruzual \& Charlot 2003) that is meant to take account of the stellar metallicities and the star formation histories. Their estimate of the stellar mass-to-light ratio is $M_{\text {stars }} / L_{z} \simeq 1.85$ for luminous galaxies, with magnitudes $M_{z}<M_{z}^{*}-0.8$, and it decreases gradually to $M_{\text {stars }} / L_{z} \simeq 0.65$ for fainter galaxies with
$M_{z} \simeq M_{z}^{*}+3$ for a galaxy sample at $z \approx 0.05$. Our estimate of the resulting luminosity-functionweighted mean is

$$
\left\langle M_{\text {stars }} / L_{z}\right\rangle \simeq 1.5 \pm 0.3
$$

for the IMF Kauffmann et al. used. Equation (22) represents the present mass in stars and stellar remnants, and it excludes the mass shed by evolving stars and returned to diffuse components.

The estimate of $M_{\text {stars }} / L$ assumes a universal IMF, which is not thought to seriously violate the observational constraints. We note, however, that a possible change of the IMF at very high redshift need not seriously affect our analysis because star formation a high redshift contributes little to the present total mass in stars. The IMF is particularly uncertain at the subsolar masses that make little contribution to the light but can make a considerable contribution to the mass. We consider two continuous broken power law models, of the form

$$
d N / d m \propto m^{-(x+1)},
$$

where, in the first model,

$$
\begin{aligned}
x & =-0.5, \quad 0.01<m<0.1 m_{\odot}, \\
& =0.25 \quad 0.1<m<1 m_{\odot}, \\
& =1.35 \quad 1<m<100 m_{\odot},
\end{aligned}
$$

and, in the second model,

$$
\begin{aligned}
x & =-0.7 \quad 0.01<m<0.08 m_{\odot}, \\
& =0.3 \quad 0.08<m<0.5 m_{\odot}, \\
& =1.3 \quad 0.5<m<100 m_{\odot} .
\end{aligned}
$$

The first line in the first model is from Burgasser et al. (2003), the second line is from Reid, Gizis, \& Hawley (2002), and the third line, for $m>1 m_{\odot}$, is the standard Salpeter (1955) IMF. The second model is from Kroupa (2001). Yet another IMF, that of Chabrier (2003), is in between these two. For our model IMF we take the Salpeter index for $m \geq 1 m_{\odot}$. It is known that the Salpeter slope gives satisfactory UBV colors and $\mathrm{H} \alpha$ equivalent widths for normal galaxies (Kennicutt 1983), whereas an IMF with a steeper slope (e.g., Scalo 1986) is not favored in this regard. The consensus seems to be that the power law index is smaller than the Salpeter value at sub-Solar masses. The two IMF given above still differ significantly at 
$m<m_{\odot}$, however, For the subsolar IMF, we take the geometric mean of the above two models after mass integration, and we take the difference as an indication of the error $( \pm 18 \%)$.

The stellar mass-to-light ratio in equation (22) assumes the IMF in equation (25). With our adopted IMF the stellar mass-to-light ratio is 1.18 times the number in equation (22). ${ }^{10}$ Thus we get our fiducial estimate,

$$
\left\langle M_{\text {star }} / L_{z}\right\rangle=1.23 \pm 0.33
$$

This translates to $M / L_{B} \approx 2.4$, or 0.7 times that used in FHP, which employed the subsolar mass IMF of Gould, Bahcall \& Flynn (1996). In the Salpeter IMF, with $x=1.35$ cut off at $0.1 m_{\odot}$, the mass-to-light ratio is 1.48 times our adopted value. The Kennicutt (1983) IMF results in 0.81 times equation (26).

The IMF at substellar masses, $m<0.08 m_{\odot}$, is more uncertain, but recent observations of $\mathrm{T}$ dwarfs in the solar neighbourhood indicate $x<0$ (e.g., Burgasser et al. 2003; 2004). In the two IMF models quoted above the substellar mass is 6 to $9 \%$ of the mass integral for $0.08<m<1 m_{\odot}$. We adopt $8 \%$ and assign an error of $50 \%$.

We estimate the mass density locked in stars, including those in dead stars, to be $\Omega_{\text {stars }}=$ $0.0024 \pm 0.0007$. A comparable estimate is derived from $b_{J}, J, K_{s}$ multicolour photometry of 2MASS combined with $2 \mathrm{dF}$ data by Cole et al. (2001), $\Omega_{\text {stars }}=0.0029 \pm 0.0004$ with the IMF we have adopted. For the energy inventory we take the mean of our present number and that of Cole et al.:

$$
\Omega_{\text {stars }}=0.0027 \pm 0.0005 \text {. }
$$

This means that the stars contain $6.0 \pm 1.3 \%$ of the total baryons. The FHP estimate is $\Omega_{\text {stars }}=$ $0.0019-0.0057$. Equation (27) also is consistent within the errors with the more recent estimates by Salucci \& Persic (1999), Kochanek et al. (2001), and Glazebrook et al. (2003), and with Shull's (2003) baryon inventory.

We attempt to partition the stars into their species. Our estimates of the mass fractions in stars on the main sequence (MS) and substellar

${ }^{10}$ The IMF are normalised so that the mass integrals between 0.9 and $2.0 m_{\odot}$ are equal. The result is virtually identical with those with the two IMFs normalised at $1 m_{\odot}$. objects (SS), and the mass fractions in stellar remnants, including white dwarfs (WD), neutron stars (NS) and stellar mass black holes (BH), are shown in Table 2. Stars on the MS are represented by the present-day mass function (PDMF), which for $1<m<100 m_{\odot}$ is constructed by multiplying the IMF by $m^{-2.5}$ to account for the lifetime of massive main sequence stars; for $m<1 m_{\odot}$ the PDMF is the same as the IMF. A more detailed estimate of the relation between PDMF and IMF is possible but not needed for our purposes because the mass fraction in stars with masses $m>m_{\odot}$ is small. We take $m=0.08 m_{\odot}$ as the mass dividing hydrogen-burning and substellar mass 'brown dwarfs' (Hayashi \& Nakano 1963; Kumar 1963) and the observational limit $0.01 m_{\odot}$ as the lower end of substellar masses.

As indicated in the third line of Table 2, we take the average mass of a white dwarf to be $\langle m\rangle=0.62 m_{\odot}$, for consistency with the model we describe in section 2.5.2 (eq. [70]). This is close to the value from recent observations, $0.604 \mathrm{~m}_{\odot}$ (Bergeron \& Holberg, in preparation; Bergeron private communication). We assume all stars with initial masses in the range $1<m<8 m_{\odot}$ end up as white dwarfs with the adopted average mass, and the rest of the mass returns to the interstellar medium. With our adopted IMF this model predicts that the ratio of masses in white dwarfs to main sequence stars is $\rho(\mathrm{WD}) / \rho(\mathrm{MS})=0.176$. This can be compared to the observations. The $2 \mathrm{dF}$ survey (Vennes et al. 2002), with the use of our mean mass $0.62 m_{\odot}$, yields a measure of the mass density of DA white dwarfs, $\rho(\mathrm{DA}-\mathrm{WD}$, local $)=(4.2 \pm 2.3) \times 10^{-3} m_{\odot} \mathrm{pc}^{-3}$. This is multiplied by 1.3 to account for $\mathrm{DB}, \mathrm{DQ}$ and DZ white dwarfs (Harris et al. 2003), to give the total white dwarf mass density $\rho(\mathrm{WD}$,local $)=$ $(5.5 \pm 3.0) \times 10^{-3} m_{\odot} \mathrm{pc}^{-3}$. This divided by the local density of main sequence stars, $\rho$ (MS,local $)=$ $0.031 \pm 0.002 m_{\odot} \mathrm{pc}^{-3}$, from Reid, Gizis, \& Hawley (2002), and $0.041 \pm 0.003 m_{\odot} \mathrm{pc}^{-3}$ from Chabrier (2003), gives $\rho(\mathrm{WD}) / \rho(\mathrm{MS})=0.16 \pm$ 0.10 , which agrees with our model prediction, albeit with a large uncertainty.

We assume all stars in the initial mass range

$$
8<m<25 m_{\odot}
$$

end up as neutron stars with mass $1.35 m_{\odot}$ (Nice, Splaver, \& Stairs 2003; Thorsett \& Chakrabarty 
TABLE 2

STAR MASS FRACTIONS

\begin{tabular}{lcccc}
\hline \hline initial mass range & fate & remnant $^{\mathrm{a}}$ & mass fraction & mass consumed $^{\mathrm{b}}$ \\
\hline $0.01<m<0.08$ & SS & $\ldots$ & 0.052 & 0.052 \\
$0.08<m<100^{\mathrm{c}}$ & MS & $\ldots$ & 0.769 & 0.769 \\
$1<m<8$ & WD & 0.62 & 0.135 & 0.463 \\
$8<m<25$ & NS & 1.35 & 0.019 & 0.186 \\
$25<m<100$ & $\mathrm{BH}$ & 7.5 & 0.025 & 0.146 \\
\hline sum & 1.0 & 1.616 \\
\hline \multicolumn{5}{l}{} \\
${ }^{\mathrm{a}}$ Mass in units of Solar masses \\
${ }^{\mathrm{b}}$ The gas consumed to make unit mass of stars now present. \\
${ }^{\mathrm{c}}$ For the mass range $1<m<100 m_{\odot}$ those stars burning today are counted.
\end{tabular}

1999), and the rest of the star mass is recycled. Estimates of the lower critical mass for stellar core collapse vary from 6 to $10 m_{\odot}$ (Reimers \& Koester 1982; Nomoto 1984; Chiosi, Bertelli, \& Bressan 1992). The upper critical mass for the formation of a neutron star is more uncertain; our choice in equation (28) is taken from Heger et al. (2003). We assume all stars with main sequence mass above the limit in equation (28) end up as stellar black hole remnants with mass $m_{\bullet}=7.5 m_{\odot}$, with the rest of the mass recycled. This again follows Heger et al. The remnant mass is quite uncertain, and Heger et al. also indicate that some stars with masses above the range in equation (28) may produce neutron stars.

The last column in Table 2 is the cumulative amount of mass that has gone into stars, including what is later shed, normalised to the mass in column (4). According to these estimates, 1.6 times the mass in observed stars was used (and reused) in star formation.

Entries 3.3 to 3.8 in Table 1 are based on the partition of $\Omega_{\text {stars }}$ among main sequence stars and star remnants in the fourth column of Table 2, with the FHP partition between the two galaxy population types in the proportion

$$
\text { spheroid }: \operatorname{disc}=0.74: 0.26
$$

of mass in ellipticals plus bulges of spirals to the mass in disks plus irregulars.

\subsubsection{Consistency with the Star Formation Rate}

We can compare our estimate of the mass in stars to the mass integrated over the star forma- tion rate density (the SFR) from high redshift to the present. The $\mathrm{H} \alpha$ luminosity density at zero redshift measured from an SDSS nearby galaxy sample is $\mathcal{L}(\mathrm{H} \alpha)=10^{39.31_{-0.08}^{+0.11}} h \mathrm{erg} \mathrm{s}^{-1} \mathrm{Mpc}^{-3}$ (Nakamura et al. 2004). This agrees with the earlier value obtained by Gallego et al. (1995). Within the star formation models Glazebrook et al. (1999) explored, $1 m_{\odot} \mathrm{yr}^{-1}$ of star formation produces $\mathrm{H} \alpha$ luminosity $L_{\alpha}=\left(2.00_{-0.24}^{+0.92}\right) \times 10^{41}$ $\mathrm{erg} \mathrm{s}^{-1}$ for our adopted IMF. The ratio of $\mathcal{L}(\mathrm{H} \alpha)$ to $L_{\alpha}$ is an estimate of the present-day SFR,

$$
\psi(0)=0.0071_{-0.0035}^{+0.0021} m_{\odot} \mathrm{yr}^{-1} \mathrm{Mpc}^{-3} .
$$

The evolution of the SFR at $z \lesssim 1$ is now observationally well determined (Lilly et al. 1996; Heavens et al. 2004; see Glazebrook et al. 1999 for a summary of the measurements up to that time), and may be approximated as

$$
\psi(t)=\psi(0) e^{\left(t_{o}-t\right) / \tau_{\mathrm{sf}}}, \quad z<0.85,
$$

where

$$
\tau_{\mathrm{sf}} \simeq 2.9 \mathrm{Gyr},
$$

and $t_{o}-t$ is the time measured back from the present in the $\Lambda$ CDM cosmology (Fukugita \& Kawasaki 2003; see also Glazebrook et al. 1999). The situation at higher redshift is still controversial (Madau et al. 1996; Steidel et al. 1999). We shall suppose that the SFR is constant from $z=0.85$ back to the start of star formation at redshift $z_{f}$, at the value

$$
\psi=0.0877 m_{\odot} \mathrm{yr}^{-1} \mathrm{Mpc}^{-3}, 0.85<z<z_{f} .
$$


In this model the integrated star formation is

$$
\begin{aligned}
& \Omega_{\text {stars }} \simeq 0.0038 \text { if } z_{f}=2 \\
& \simeq 0.0047 \text { if } z_{f}=3, \\
& \simeq 0.0053 \text { if } z_{f}=5
\end{aligned}
$$

There is a significant downward uncertainty, $\delta \Omega_{\text {stars }} \sim-0.0025$, arising from the uncertainty in the star formation rate (eq.[30]). Equation (27) and Table 2 indicate that the mass processed into stars, including what was later shed, is $\Omega=0.0027 \times 1.62=0.0044$. This is consistent with the cumulative star formation in equation (34). There would be a problem with the numbers if there were reason to believe that the SFR continued to increase with increasing redshift well beyond $z=1$. A constant or declining SFR at $z>1$ is well accommodated with our estimates for the baryon budget and current ideas about the star formation history at high redshift.

The star formation history determines the average reciprocal redshift factor (as in eq. [102]) for the effect of redshift on the integrated comoving energy density of electromagnetic radiation (and other forms of relativistic mass) generated by stars. In our model for the SFR the correction factor is

$$
\begin{aligned}
\left\langle(1+z)^{-1}\right\rangle^{-1} & =\frac{\int d t \mathcal{L}}{\int d t \mathcal{L} /(1+z)} \\
& =2.0 \pm 0.15
\end{aligned}
$$

where $\mathcal{L}(t)$ is the bolometric luminosity density per comoving volume, which we are assuming is proportional to the star formation rate density. The numerical value assumes the redshift cutoff is in the range $z_{f}=2$ to 5 . For the purpose of comparison of the accumulation of stellar products to the present rate of production, another useful quantity represents the integrated comoving density of energy radiated in terms of the effective time span normalised by the present-day luminosity density,

$$
\Delta t_{\text {eff }}=\mathcal{L}\left(t_{0}\right)^{-1} \int d t \mathcal{L}(t)=85 \pm 13 \mathrm{Gyr},
$$

The value assume the range of models for the star formation history in equation (34).

We can compare the rate of stellar core collapse in our model to observations of the supernova rate.
(See Fukugita \& Kawasaki 2003 and Madau, della Valle \& Panagia 1998 for similar analyses.) The present SFR in equation (30) and the critical minimum mass $8 m_{\odot}$ in equation (28) imply that the present rate of formation of neutron stars and stellar black holes is expected to be

$$
\begin{aligned}
R & =\frac{\int_{8}^{100} d m d N / d m}{\int_{0.08}^{100} d m m d N / d m} \\
& =0.0079_{-0.0039}^{+0.0024}(100 \mathrm{yr})^{-1} \mathrm{Mpc}^{-3} .
\end{aligned}
$$

Our estimate of the observed supernova rate is

$$
R_{\mathrm{SN}}=0.0076_{-0.0020}^{+0.0064}(100 \mathrm{yr})^{-1} \mathrm{Mpc}^{-3} .
$$

This is the geometrical mean of the rates from three surveys for Type II and Type Ib/c supernovae, 0.037, 0.018 and 0.017, in units of $h^{3}(100 y r)^{-1} \mathrm{Mpc}^{-3}$ (Tammann, Löffler \& Schröder 1994; Cappellaro et al. 1997; van den Bergh \& McClure 1994; see also Cappellaro, Evans \& Turatto 1999). We conclude that, if most stars with initial masses greater than about $8 m_{\odot}$ produced Type II and Ib/c supernovae, then our model for the star formation history would pass this consistency check.

We remark that in our model the comoving number density of supernovae of types II and Ib/c integrated back to the start of star formation is

$$
R \Delta t_{\text {eff }}=7 \pm 3 \times 10^{6} \mathrm{Mpc}^{-3},
$$

where the effective time span is given in equation (36).

\subsubsection{Planets and Condensed Matter}

The mass in planets that are gravitationally bound to stars must be small, but it is of particular interest to us as residents of a planet. Marcy (private communication; see also Marcy \& Butler 2000) finds that about $6.5 \%$ of nearby FGKM stars have detected Jovian-like planets, and that an extrapolation to planets at larger orbital radii might be expected to roughly double this number. In our model for the PDMF the ratio of the number density of stars in the mass range 0.08 to $1.6 \mathrm{~m}_{\odot}$ to the mass density in stars is $n / \rho=2.1 m_{\odot}^{-1}$. The product of this quantity with the mass density in stars (eq. [27]), the fraction 0.13 , and the ratio of the mass of Jupiter to the Solar mass is

$$
\Omega_{\text {planets }}=10^{-6.1} \text {. }
$$


Marcy indicates that stars with lower metallicity have fewer planets, but that may not introduce a serious error because there are fewer low metallicity stars.

There is a population of interstellar planets that have escaped from stars that have rapidly shed considerable mass. If the number of planets per star is independent of the stellar mass then, in our IMF, stars in the mass range 1 to $1.6 m_{\odot}$ had 0.025 times the number of stars that are bound to main sequence stars. Among them about $2 / 3$ are likely to be swallowed by the host stars in their giant star phases. This leaves the mass in liberated planets, and those still bound to white dwarfs, at about 0.07 times the mass density in equation (40). Planets associated with more massive stars may more likely escape during core collapse, but the numbers of stars, and perhaps planets, is small. The large uncertainty in equation (40) is whether the stellar neighborhood is a fair sample. This leads us to enter the order of magnitude in Table 1.

We consider separately the mass in objects small enough to be held together by molecular binding energy rather than gravity. The dominant amount of the former is interstellar dust. It is known (Draine 2003) that $\gtrsim 90 \%$ of silicon atoms in interstellar matter are condensed into grains, probably dominantly in the forms of enstatite $\left(\mathrm{MgSiO}_{3}\right)$ and forsterite $\left(\mathrm{Mg}_{2} \mathrm{SiO}_{4}\right)$ with the former likely three to four times more abundant (Molster et al. 2002). This means the mass in this form is $Z$ (silicates) $Z=0.17$ times the mass in heavy elements. Draine (2003) suspects that the dominant contribution to the carbonaceous material is in the form of polycyclic aromatic hydrocarbon with the inferred abundance $\mathrm{C} / \mathrm{H}=6 \times 10^{-5}$. Adding this to the silicates, we find that the mass fraction becomes $Z$ (dust) $/ Z=0.20$. The formation of dust with iron or other forms of carbonates could increase this number. Entry 3.12, $\Omega_{\mathrm{dust}}=10^{-5.6}$, is the product of the density parameter in cool gas (entries 3.9 plus 3.10) with the mean metallicity discussed below (and displayed as eq. [88]) and the 20 percent heavy element mass fraction in dust. Our estimate, which assumes that what we know about the Milky Way applies to other galaxies, is crude, but it seems likely that the mass in dust exceeds the mass in planets.

There are larger objects in which molecular binding is important. The gravitational binding energy of a roughly spherical object with mass $m$ and radius $r$ is $\sim G m^{2} / r$, and the molecular binding energy is roughly $1 \mathrm{eV}$ per atom. The molecular binding energy is larger than the gravitational energy when

$$
m \lesssim 1.2 \times 10^{28} \mathrm{~g}\left(5 \mathrm{~g} \mathrm{~cm}^{-3} / \rho\right)^{1 / 2} / \bar{A},
$$

where the mass density is $\rho$ and the mean atomic weight is $\bar{A}$. For silicates, this bound is $m=$ $8 \times 10^{26} \mathrm{~g}$. That is, gravitational binding energy dominates in the Earth and molecular binding energy dominates in the Moon and asteroids. Trujillo, Jewitt, \& Luu (2001) estimate that the mass in the Kuiper Belt objects is about a tenth of an Earth mass, or $10^{-6.5} m_{\odot}$. A comparable mass is in the moons in the Solar system. If this mass fraction were common to all stars the density parameter in these objects would be

$$
\Omega_{\text {asteroid }} \sim 10^{-9},
$$

a small fraction of the mass in dust.

\subsubsection{Neutral Gas}

The recent blind HI surveys are a significant advance over the data used by FHP to estimate the mass density in neutral atomic gas. The largest survey, HIPASS, with 1000 galaxies (Zwaan et al. 2003), gives

$$
\Omega_{\mathrm{HI}}=4.2 \pm 0.7 \times 10^{-4} .
$$

The increase over the value quoted in FHP (1.5 times the upper end value of FHP) illustrates the advantage of blind surveys over observations of programmed galaxies. The molecular hydrogen abundance from the CO survey of Keres, Yun \& Young (2003) is

$$
\Omega_{\mathrm{H}_{2}}=1.6 \pm 0.6 \times 10^{-4} .
$$

The sum of these two values is multiplied by 1.38 to accommodate helium.

We place in entry 3.9 the atomic hydrogen and the helium abundance belonging to atomic and molecular hydrogen. Entry 3.10 is the molecular hydrogen component. The mass in this neutral gas is $1.7 \pm 0.4 \%$ of the total baryon mass. 


\subsubsection{Intracluster Plasma}

The estimate of the plasma mass in rich clusters of galaxies depends on a convention for the cluster radii and masses. We use the mass $M_{200}$ contained by the nominal virial radius, $r_{200}$. This definition is more faithful to a physical definition of the part of a cluster that is close to dynamical equilibrium, and it also traces the $\mathrm{X}$-ray radius which is the definition of our hot gas. In the limiting isothermal sphere model the relation to the mass within the Abell radius $r_{A}=1.5 h^{-1} \mathrm{Mpc}$ is

$$
M_{A}=M_{o}^{1 / 3} M_{200}^{2 / 3}, \quad M_{o}=1.1 \times 10^{15} m_{\odot} .
$$

This agrees with the estimates of the two masses given by Reiprich \& Böhringer (2002), Table 4. We adopt the Reiprich \& Böhringer cluster mass density parameter,

$$
\Omega_{\mathrm{cl}}=0.012_{-0.04}^{+0.03}
$$

for the cluster mass limit

$$
M_{200}>5 \times 10^{13} m_{\odot}, \quad M_{A}>1.3 \times 10^{14} m_{\odot} .
$$

That is, $4 \%$ of the mass is assembled in rich clusters. The Bahcall \& Cen (1993) Abell mass function is consistent with the Reiprich \& Böhringer estimate. We caution that the Bahcall et al. (2003) SDSS mass function is about half what we adopt, perhaps because SDSS samples a subset of the clusters. Note also that the integral over the cluster mass within the Abell radius gives a substantially larger value for $\Omega_{\mathrm{cl}}$ because $M_{A}>M_{o}$ at the masses $M \gtrsim 10^{14} m_{\odot}$ that dominate the integral for a given mass function.

The abundance of hot baryons in clusters is obtained from the expression

$$
\begin{aligned}
\Omega_{\mathrm{b}, \mathrm{cl}} & =0.012\left(\Omega_{\mathrm{b}}-\Omega_{\text {stars }}\right) / \Omega_{m} \\
& =0.0018 \pm 0.0007
\end{aligned}
$$

The change from the value in FHP is mainly due to the definition of the cluster mass, as also noted by Reiprich \& Böhringer.

\subsubsection{Massive Black holes}

We follow the standard idea that the massive objects in the centers of galaxies are black holes that formed by the accretion of baryons. Baryons entering black holes are said to lose their identity, but for our accounting it is appropriate to consider them to be sequestered baryons.

We define the characteristic efficiency factor $\epsilon_{n}$ for black hole formation (where the subscript is meant to distinguish the massive black holes in the centers of galaxies from stellar mass black holes) by the black hole mass produced out of an initial diffuse baryon mass $m_{\mathrm{b}}$,

$$
m_{\mathrm{bh}}=\left(1-\epsilon_{n}\right) m_{\mathrm{b}}
$$

where the energy released in electromagnetic radiation, neutrinos, kinetic energy, and possibly gravitatonal radiation is

$$
m_{\mathrm{emitted}}=\epsilon_{n} m_{\mathrm{b}}
$$

The baryon mass sequestered in massive black holes, $m_{\mathrm{b}}=m_{\mathrm{bh}} /\left(1-\epsilon_{n}\right)$, could be substantial if $\epsilon_{n}$ were close to unity. However, if an appreciable part of the binding energy were released as electromagnetic radiation then the bounds on the radiation background (in category 7 ) and those of the CMBR distortion (eq. [15]) would require that the energy was released at very high redshift, $k T>10^{7} \mathrm{~K}$. The estimates discussed in $\S 2.7 .6$ indicate the mass fraction released in gravitational radiation is small. Thus it seems likely that $\epsilon_{n}$ is small, as is assumed in entry 3.13. The efficiency factor $\epsilon_{s}$ typical of stellar mass black holes does not appear in entry 3.7, because this estimate is based on an analysis of the progenitor star masses. The estimate of the mean mass density in massive black holes that is used for entry 3.13 is discussed in $\S 2.5 .3$.

\subsubsection{Intergalactic Plasma}

Entry 3.1, for the baryon mass outside galaxies and clusters of galaxies, is the difference between our adopted value of the baryon density parameter (eq. [17]) and the sum of all the other entries in category 3. Within standard pictures of structure formation this component could not be in a compact form such as planets, but rather must be a plasma, diffuse enough to be ionized by the intergalactic radiation or else shocked to a temperature high enough for collisional ionization, but not dense and hot enough to be a detectable X-ray source outside clusters and hot groups of galaxies.

In our baryon budget $90 \%$ of the baryons are in this intergalactic plasma. It is observed in several 
states. Quasar absorption lines show matter in low and high atomic ionization states in the halos of $L \sim L_{*}$ galaxies, extending to radii $\sim 200 \mathrm{kpc}$ (Chen et al. 2001 and references therein). Absorption lines of HI and MgII reveal low surface density photoionized plasma at kinetic temperature $T \sim 10^{4} \mathrm{~K}$, which can be well away from $L \sim L_{*}$ galaxies, as discussed by Churchill, Vogt, \& Charlton (2003), and Penton, Stocke \& Shull (2004). The latter authors estimate that $30 \%$ of the baryons are in this state. Absorption lines of O VI around galaxies and groups of galaxies (Tripp, Savage \& Jenkins 2000; Sembach et al. 2003; Shull, Tumlinson, \& Giroux 2003; Richter et al. 2003), reveal matter that may be excited by photoionization by the $\mathrm{X}$ ray background radiation and by collisions in plasma at the kinetic temperature $T \sim 10^{6} \mathrm{~K}$ characteristic of the motion of matter around galaxies (Cen et al. 2001). The detection of O VII and O VIII absorption lines also indicates the presence of higher temperature regions in the local IGM (Fang et al. 2002; Fang, Sembach \& Canizares 2003). Improvements in the constraints on the amount of matter in these various states of intergalactic baryons will be followed with interest.

For the inventory we adopt the measure of the concentration of dark matter around galaxies in equation (13), and the argument discussed in $\S 2.3 .8$ that the baryons are distributed like the dark matter on scales comparable to the virial radii of galaxies. The resulting division into the mass in baryons near the virial radii of normal galaxies outside clusters (entry 3.1a), and the mass well away from galaxies and compact groups and clusters of galaxies (entry 3.1b), is presented as sub-components, because the sum is much better constrained than the individual values.

\subsubsection{Baryon Cooling}

We comment here on a simple picture for the cooling and settling of baryons onto galaxies. The sum of the baryon mass densities belonging to galaxies, in entries 3.3 to 3.13 , is $\Omega_{\mathrm{b}, \mathrm{g}}=0.0035$. This is $8 \%$ of the total baryon mass. Suppose $\Omega_{\mathrm{b}, \mathrm{g}}$ consists of all baryons gathered from radius $r_{g}$ around $L \sim L_{*}$ galaxies, and suppose we can neglect the addition of baryons by settling from further out and the loss by galactic winds. That is, we are supposing that at $r>r_{g}$ the ratio of the baryon density to the dark matter density is the cosmic mean value, and that the baryons closer in have collapsed onto the galaxies. In this picture the characteristic radius of assembly of the baryons satisfies

$$
\frac{2 n_{g} r_{g} \sigma^{2}}{G \rho_{m}}=\frac{\Omega_{\mathrm{b}, \mathrm{g}}}{\Omega_{\mathrm{b}, \text { total }}},
$$

in the limiting isothermal sphere approximation (eq. [9]).

In equation (51) $n_{g}$ is a measure of the number density of luminous galaxies. We record here our choices for this quantity and related parameters that are used elsewhere. We take

$$
n_{g}=\mathcal{L}_{r} / L_{r}^{*}=0.017 h^{3} \mathrm{Mpc}^{-3}
$$

where $\mathcal{L}_{r}$ is the luminosity density (eq. [14]). The characteristic galaxy luminosity,

$$
\begin{aligned}
& L_{B}^{*}=1.07 \times 10^{10} h^{-2} L_{\odot}, \\
& L_{r}^{*}=1.45 \times 10^{10} h^{-2} L_{\odot}, \\
& L_{z}^{*}=2.37 \times 10^{10} h^{-2} L_{\odot},
\end{aligned}
$$

is the luminosity parameter in the Schechter function, with the power law index $\alpha_{B}=-1.1, \alpha_{r}=$ -1.13 , and $\alpha_{z}=-1.14$. We refer some estimates of energy densities to what is known about the Milky Way, for which we need the effective number density of Milky Way galaxies. In the B band the Milky Way luminosity is $L_{\mathrm{MW}}=1.3 L_{B}^{*}$, and the effective number density is

$$
n_{\mathrm{MW}}=\mathcal{L}_{B} / L_{\mathrm{MW}}^{*}=0.013 h^{3}(\mathrm{Mpc})^{-3} .
$$

Almost the same density follows when referred to the r-band. The Milky Way parameters are discussed in the Appendix.

With the characteristic velocity dispersion in equation (10) and the characteristic galaxy number density in equation (52) the baryon accretion radius defined by equation (51) is

$$
r_{g} \simeq 30 h^{-1} \mathrm{kpc}
$$

at density contrast $1.3 \times 10^{4}$ and plasma density $n_{\text {gas }}\left(r_{g}\right) \sim 0.007 h^{3} \mathrm{~cm}^{-3}$. If plasma at this radius were supported by pressure at the onedimensional velocity dispersion $\sigma \simeq 160 \mathrm{~km} \mathrm{~s}^{-1}$ in equation (10) the temperature would be $T \simeq$ $2 \times 10^{6} \mathrm{~K}$. At this density and temperature the 
thermal bremsstrahlung cooling time would be short enough, $\sim 4 \times 10^{9} \mathrm{yr}$, that stars would have formed and disks matured at $z \sim 1$.

We have lower bounds on the cooling radius from the observation that the neutral atomic hydrogen density around $L_{*}$ galaxies reaches $N_{\mathrm{HI}}=$ $1.8 \times 10^{20} \mathrm{~cm}^{-2}$ at the effective radius $\sim 20 h^{-1} \mathrm{kpc}$ (Bosma 1981), and Mg II absorption lines are observed at radius $\sim 40 h^{-1} \mathrm{kpc}$ (Steidel, Dickinson \& Persson 1994). For the Milky Way the distribution of RR Lyr stars cuts off sharply at $50 \mathrm{kpc}$ (Ivezić et al. 2000). The cooling radius must be larger than these indicators of relatively cool matter. Equation (55) is not inconsistent with this condition. Though the history of baryon accretion by galaxies undoubtedly is complex, we can imagine, as a first approximation, that a substantial fraction of the baryons now concentrated in galaxies are there because they were able to cool and settle from an initial distribution similar to that of the dark matter.

The relative distributions of baryons and dark matter at distances much larger than $r_{g}$ from galaxies might not be greatly disturbed from the primeval condition. If so, then the product of the baryon density parameter with the virialized dark matter mass fraction in equation (13) is an estimate of the baryon mass that resides within the virial radii of normal galaxies, and the remainder,

$$
\Omega_{\mathrm{b}, \mathrm{ig}} \sim 0.016
$$

which is presented as entry $3.1 \mathrm{~b}$, would be located outside galaxies and remain less than fully documented.

\subsection{Primeval Gravitational Energy}

In the $\Lambda$ CDM cosmology the gravitational binding energy of the present mass distribution has two contributions. The first, which we term primeval, is a result of the purely gravitational growth of mass fluctuations out of the small adiabatic departures from a homogeneous mass distribution present in the initial conditions for the FriedmannLemaitre cosmology. The second, to be discussed in the next subsection, is the result of dissipative settling of baryons that produced the baryondominated luminous parts of the galaxies along with stars and star remnants. We can find sensible approximations to the primeval and dissipative components because, as we will discuss, the characteristic length scales are well separated.

The primeval gravitational energy is defined by imagining a universe with initial conditions identical to ours in all respects except that the baryonic matter in our universe is replaced by an equal mass of $\mathrm{CDM}$ in the reference model. At the present world time this reference model contains a clustered distribution of massive halos with gravitational binding energy density that we term the primeval component. ${ }^{11}$ This component is estimated as follows.

The Layzer (1963) - Irvine (1961) equation for the evolution of the kinetic and gravitational energies of nonrelativistic matter, such as CDM, that interacts only by gravity is

$$
\frac{d}{d t}(K+W)+\frac{\dot{a}}{a}(2 K+W)=0 .
$$

The kinetic energy per unit mass is

$$
K=\left\langle m \vec{v}^{2} / 2\right\rangle /\langle m\rangle,
$$

where $\vec{v}$ is the peculiar velocity of a particle with mass $m$. The gravitational potential energy per unit mass is

$$
W=-\frac{1}{2} G \rho_{m} \int d^{3} r \xi(r) / r
$$

where $\rho_{m}$ is the mean mass density and $\xi$ is the reduced mass autocorrelation function.

We can use a simple limiting case of the LayzerIrvine equation, because in the $\Lambda \mathrm{CDM}$ cosmology

\footnotetext{
${ }^{11}$ One surely would say that in this model universe the virialized dark matter halos have gravitational binding energy. Since there was no energy transfer to some other form, one might also want to say that this binding energy must have been present in the initial conditions. Furthermore, one can assign to a linear mass density fluctuation with contrast $\delta(t)>0$, and comoving radius $x$ (physical radius $x a(t)$ ), a gravitational energy per unit mass, $W^{\prime} \sim-G\langle\rho\rangle \delta(a x)^{2}$, which is constant in linear perturbation theory and comparable to the binding energy of the final virialized halo. Perhaps one can use this as a guide to a definition of the primeval energy belonging to the density fluctuation, despite the problem that the mean of $W^{\prime}$ vanishes, and the fact that in general relativity theory there is no general definition of the global energy density of a statistically homogeneous system. We have not been able to find a useful approach along these lines. We might add that if our universe had been Einstein-de Sitter then we would have defined the primeval energy density by a numerical solution of equation (57).
} 
the universe has now entered $\Lambda$-dominated expansion, which has caused a significant suppression of the rate of growth of large-scale departures from homogeneity. This means that the first term in equation (57) has become small compared to the second term. Thus it is a reasonable approximation to take $2 K=-W$, the usual virial equilibrium relation. Then the gravitational binding energy per unit mass is $U=K+W=W / 2$, and the cosmic mean primeval gravitational binding energy is the product of $U$ with the mean mass density $\Omega_{m}$ in matter (eq. [8]). With the normalization $P(k)=\int d^{3} r \xi(r) e^{i \vec{k} \cdot \vec{r}}$ for the mass fluctuation power spectrum, the expression for the primeval gravitational binding energy in this approximation is

$$
\Omega_{\mathrm{BE}, \mathrm{p}}=-\frac{3 \Omega_{m}^{2} H_{o}^{2}}{16 \pi^{2}} \int_{o}^{\infty} d k P(k) .
$$

For a numerical value we use the mass fluctuation power spectrum $P(k)$ in Figure 37 of Tegmark et al. (2004b) at $k<0.1 h \mathrm{Mpc}^{-1}$. At smaller scales the Tegmark et al. spectrum decreases too rapidly with increasing wavenumber $k$ to be a good approximation to the present mass distribution (Davis \& Peebles 1983). We approximate the spectrum as

$$
P(k)=7000\left(\frac{0.1 h \mathrm{Mpc}^{-1}}{k}\right)^{1.23} h^{-3} \mathrm{Mpc}^{3},
$$

at $k>0.1 h \mathrm{Mpc}^{-1}$. This is based on the Fourier transform of the pure power law model for the galaxy autocorrelation function (eq. [5]),

$$
P=4 \pi r_{o}^{\gamma} k^{\gamma-3} \Gamma(2-\gamma) \sin \pi(2-\gamma) / 2,
$$

which gives $P=5200 h^{-3} \mathrm{Mpc}^{3}$ at $k=0.1 h \mathrm{Mpc}^{-1}$. We choose the somewhat larger normalization in equation (61) to fit the SDSS measurement. The numerical result is

$$
\int_{o}^{\infty} P(k) d k=4800 h^{-2} \mathrm{Mpc}^{2} .
$$

The integral of the Tegmark et al. (2004b) power spectrum over all wavenumbers is $2700 h^{-2} \mathrm{Mpc}$, which is 0.55 times the value in equation (63). Our estimate of the integral is based on measurements of the actual power spectrum, not the spectrum that would have obtained if there were no dissipative settling of baryons, but the error is small because the integral is dominated by lengths large compared to the scale of separation of baryons from the dark matter.

Equations (60) and (63) yield the entry in line 4 of Table 1 ,

$$
\Omega_{\text {grav }, \mathrm{p}}=-7.7 \times 10^{-7} .
$$

This is the density parameter of the gravitational binding energy of the present departure from a homogeneous mass distribution, ignoring the effects of the dissipative settling of baryons. It will be useful to note that a measure of the length scale of the gravitational energy is the half-point of the integral in equation (63), at wavenumber $k_{1 / 2}=0.27 h \mathrm{Mpc}^{-1}$, or half wavelength

$$
\lambda_{1 / 2}=\pi / k_{1 / 2}=12 h^{-1} \mathrm{Mpc} .
$$

The kinetic energy per unit mass belonging to equation (64) is

$$
K=-U=3\left(410 \mathrm{~km} \mathrm{~s}^{-1}\right)^{2} / 2 .
$$

The velocity in parentheses is the one-dimensional single-particle line-of sight rms peculiar velocity.

The gravitational binding energy is not equal to a sum over the contributions from individual objects, but we can write useful approximations to the decomposition into the three components the virialized parts of the massive halos of $L \sim L_{*}$ galaxies, the rich clusters, and large-scale clustering - shown in category 4 in the inventory.

Since galaxy rotation curves tend to be close to flat we write the binding energy of the virialized parts of the dark matter halos of the galaxies as

$$
\Omega_{\mathrm{grav}}=-\frac{3 \Omega_{m} \sigma^{2}}{2} \frac{\rho\left(r_{v}\right)}{\rho_{m}}=10^{-7.2}
$$

where $\Omega_{m}$ is the matter density parameter (eq. [8]), $\sigma$ is the characteristic velocity dispersion in equation (10) and the last factor is the virialized mass fraction (eq. 13). This is about $10 \%$ of the total gravitational binding energy. The dissipative settling that produced the baryon-dominated luminous parts of the galaxies would have perturbed the massive halos, but the disturbance to the primeval gravitational energy is small because the baryon mass fraction is small.

Our estimate of the binding energy of the dark halos of the galaxies may be compared to the density parameter given by equation (60) when the 
integral over $P(k)$ is restricted to small scales, $k>\pi / r_{v}$, where $r_{v}$ is the virial radius in in equations (10) and (67). The result, with equation (61) for $P(k)$, is twice the value in equation (67). The difference is an indication of the ambiguity of separating gravitational energy into components. For entry 4.1 we adopt the value in equation (67) as the more directly interpretable.

Equation (67) is a reasonable approximation for most of the mass in galaxy-size dark halos in luminous field galaxies such as the Milky Way, but in rich clusters the galaxies tend to share a dark halo that is close to smoothly distributed across the cluster. Our estimate in entry 4.2 for the primeval gravitational binding energy belonging to rich clusters follows equation (67), with $\sigma=800$ $\mathrm{km} \mathrm{s}^{-1}$ and $\Omega_{\mathrm{cl}}$ from equation (46). Rich clusters share about $15 \%$ of the total gravitational binding energy.

Entry 4.3 is the difference between the total in entry 4 and the sum of entries 4.1 and 4.2. The Layzer-Irvine equation indicates that the binding energy is dominated by a length scale (eq. [65]) that is much larger than galaxy virial radii. Consistent with this, entry 4.3 is larger than entry 4.1 for the binding energy of the dark halos of galaxies. The difference is not large, however, because at small scales the integral over the power spectrum converges slowly, as $k^{-0.23}$.

\subsection{Dissipative Gravitational Settling}

Dissipative settling has increased the magnitude of the gravitational binding energy from that prescribed by the primeval conditions considered in the last section. In $\S 2.5 .1$ we discuss the energy released in producing the increased mean density of baryons relative to dark matter in the luminous parts of the galaxies, in $\S 2.5 .2$ we estimate the gravitational energy released in stellar formation and evolution, and in $\S 2.5 .3$ we consider the central massive compact objects in galaxies.

\subsubsection{The Luminous Parts of Galaxies}

In the Milky Way galaxy the mass within our position, at about $8 \mathrm{kpc}$ from the center, is roughly equal parts baryonic and dark matter, or about 6 times the cosmic mean ratio (eq. [19]). This is thought to be the usual situation in the luminous parts of normal galaxies. The amount of gravi- tational binding energy released in producing this concentration of baryons depends on how it was done. In one limiting case one may imagine that stars formed in the centers of low mass dark halos with relatively small dissipation of energy - apart from star formation - because the depths of the gravitational potential wells were small, and that the low mass halos later merged without any additional dissipation, the dense baryon-dominated parts remaining near the densest regions to form the present-day baryon-dominated luminous parts of galaxies. (This is an extreme version of the scenario discussed by Gao et al. 2003). In another extreme, one may imagine that the baryons settled into previously assembled galaxy-scale halos, which would dissipate considerably more energy. A galaxy has a definite computable gravitational binding energy, of course (apart from the difficulty of correcting for ongoing accretion), but to relate this to the energy dissipated in producing the galaxy would require an analysis of what the mass distribution would have been in the absence of dissipation, which is not an easy task.

These considerations lead us to offer only a crude estimate for entry 5.1, which we write as the product of the density parameter belonging to baryons in galaxies - the sum $\Omega_{\mathrm{b}, \mathrm{g}}=0.0035$ of the density parameters in entries 3.3 to 3.13 with the kinetic energy per unit mass, $K=3 \sigma^{2} / 2$ and $\sigma=160 \mathrm{~km} \mathrm{~s}^{-1}$. The result is a $2 \%$ addition to the primeval halo gravitational binding energy (entry 4.1). If the baryon concentrations in galaxies formed at high redshifts in small halos the dissipative energy released would be an even smaller fraction of the total.

\subsubsection{Stellar Binding Energy}

The amount of binding energy released in star formation is easy to define because the relative length scale is small. We write the gravitational binding energy per unit mass for a star with mass $m$ and radius $r$ as

$$
\frac{\mathrm{BE}}{m}=-K \frac{G m}{r c^{2}} .
$$

The prefactor for the Sun is $K_{\odot}=1.74$, and $K=$ 0.3 for a homogeneous sphere.

For main sequence stars we use the zero-age mass-radius relation, $r \simeq 0.85 \mathrm{~m}^{0.80}$ for $0.08<$ $m<0.79, r \simeq 0.93 m^{1.17}$ for $0.79<m<1.38$, 
and $r \simeq 1.15 m^{0.52}$ for $1.38<m<100$, in solar units. These numbers are assembled from Ezer \& Cameron (1967), Cox \& Giulli (1968) and Cox (2000). Integration of $G m^{2} / r$ over the PDMF gives $\mathrm{BE} / m=3.7 \times 10^{-6}$. The product of the last number with the density parameter of the mass in main sequence stars (entries 3.3 plus 3.4 ), with $K \simeq K_{\odot}$, is the estimate of the gravitational binding energy, $\Omega_{\mathrm{BE}}=-10^{-8.1}$, for stars. We similarly obtain the substellar gravitational binding energy, $\Omega_{\mathrm{BE}}=-10^{-9.6}$, where $r$ is fixed at $0.096 r_{\odot}$ (Burrows et al. 2001). This is a small addition to the sum in entry 5.2 .

We construct a model for the white dwarf mass function from an approximation to the relation between the progenitor main sequence mass and the white dwarf remnant mass (Claver et al. 2001; Weidemann 2000),

$$
m_{\mathrm{wd}}=0.08 m_{\mathrm{ms}}+0.45 m_{\odot},
$$

and our IMF. White dwarf masses run from 0.53 to $1.09 m_{\odot}$ for the main sequence mass range $1<m_{\mathrm{ms}}<8 m_{\odot}$ we have adopted. The white dwarf mass function $d N / d m_{\mathrm{wd}}=$ $\left(d N / d m_{\mathrm{ms}}\right)\left(d m_{\mathrm{ms}} / d m_{\mathrm{wd}}\right)$ thus obtained agrees well with the observed white dwarf mass distribution of Bergeron \& Holberg for $m \gtrsim 0.5 m_{\odot}$ (in preparation). Here we ignore low mass helium core white dwarfs. In our mass function the mean white dwarf mass is

$$
\left\langle m_{\mathrm{wd}}\right\rangle=0.62 m_{\odot} .
$$

From our mass function and the mass-radius relation given by Shapiro and Teukolsky (1983) we obtain the mean white dwarf gravitational binding energy per unit mass,

$$
\frac{\mathrm{BE}}{m}=-58 K_{\mathrm{wd}} \frac{G m_{\odot}}{r_{\odot} c^{2}}=-1.2 \times 10^{-4} .
$$

Since the fractional half-mass radius of a white dwarf is 0.57 times the solar value (Schwarzschild 1958), we have taken $K_{\mathrm{wd}} \simeq 1.0$. The product with the mass density in white dwarfs (entry 3.5 ) is entry 5.3 .

We take the binding energy of a neutron star to be $3 \times 10^{53}$ erg (e.g., Burrows 1990; Janka \& Hillebrandt 1989), or

$$
\mathrm{BE} / m=-0.12 \text {. }
$$

The product with entry 3.6 is entry $5.4, \Omega_{\mathrm{BE}, \mathrm{NS}}=$ $10^{-5.2}$. The gravitational binding energies in neutron stars and stellar mass black holes are substantially larger than the gravitational binding energies in all other forms.

\subsubsection{Black Hole Binding Energy}

Our definition of the binding energy associated with a black hole requires careful explanation because it has some curious properties, including violation of the thought that it would be logical to consider the mass of a black hole to be purely gravitational if the matter out of which it formed has lost its existence.

We choose the definition by analogy to nuclear and Newtonian gravitational binding energy, in terms of the energy liberated in the assembly of a system out of its initial parts, that is, the difference between the total mass of the initial parts and the mass of the assembled system. In the same way, we use equations (49) and (50) to define the binding energy of a black hole by the difference between the mass $m_{\mathrm{b}}$ of the initial parts - baryons - and the mass $m_{\mathrm{bh}}=\left(1-\epsilon_{n}\right) m_{\mathrm{b}}$ of the final black hole. Thus our definition of the binding energy of a black hole is

$$
\mathrm{BE}=-\epsilon_{n} m_{\mathrm{b}}=-\frac{\epsilon_{n}}{1-\epsilon_{n}} m_{\mathrm{bh}} .
$$

The magnitude of BE is the energy emitted as electromagnetic and gravitational radiation, neutrinos, and kinetic energy, as is appropriate for our purpose of telling the energy transfers and balancing the baryon budget. It will be noted that in this definition the binding energy depends on how the black hole formed. For example, a Solar mass black hole that formed with efficiency $\epsilon=0.99$ is assigned binding energy $-99 m_{\odot}$, because it released that much energy, while an identical black hole that formed with $\epsilon_{n}=0.01$ is assigned a very different binding energy, $-0.01 m_{\odot}$.

Entry 5.5 for the gravitational binding energy of stellar mass black holes is the product of entry 3.7 , which is our estimate of the baryonic mass entering the black hole, with the efficiency factor $\epsilon_{s}$. In the standard picture for the formation of a stellar mass black hole, a core of baryons is first burned to heavy elements, and the subsequent collapse to a black hole may release little more energy. In this case the efficiency factor could be as 
small as $\epsilon_{s} \sim 0.009$, which is the binding energy released as starlight. It could also be as large as $\epsilon_{s} \sim 0.03$ if the collapse proceeded through a protoneutron star as an intermediate state. It cannot be much larger, however, without violating the constraints from the radiation energy density (see §2.7) and the relic supernova neutrino flux at Super-Kamiokande (Fukugita \& Kawasaki 2003).

One way to estimate the mass density in the massive black holes in the nuclei of galaxies uses the correlation of the black hole mass with the bulge luminosity. A convenient approximation to the relation, for B-band luminosities, is (Gebhardt et al. 2000; Ferrarese 2002; see also Kormendy \& Richstone 1995)

$$
M_{\bullet} / m_{\odot}=10^{-2.0 \pm 0.3} L_{\text {bulge }} / L_{\odot} .
$$

FHP estimate that the fraction of the B-band luminosity density in ellipticals and S0 galaxies is 0.24 , and the fraction in the bulges of spheroids is 0.14 . The products of equation (74) with the luminosity fractions and the luminosity density in equation (14) gives the mass density parameters in massive black holes,

$$
\begin{aligned}
& \Omega_{\bullet}(\text { early })=10^{-5.6 \pm 0.3}, \\
& \Omega_{\bullet}(\text { late })=10^{-5.9 \pm 0.3} .
\end{aligned}
$$

Salucci et al. (1999) give a consistent, but slightly larger value.

For early-type galaxies we can use the tight relation between the black hole mass and the bulge or spheroid velocity dispersion (Merritt \& Ferrarese 2001; Tremaine et al. 2002). The Sheth et al. (2003) estimate of the velocity dispersion function for early-type galaxies is

$$
d N=\phi_{*}\left(\frac{\sigma}{\sigma_{*}}\right)^{\alpha} \frac{\beta}{\Gamma(\alpha / \beta)} \frac{d \sigma}{\sigma} e^{-\left(\sigma / \sigma_{*}\right)^{\beta}},
$$

with $\alpha=6.5, \beta=1.93, \sigma_{*}=89 \mathrm{~km} \mathrm{~s}^{-1}$, $\phi_{*}=0.0020 \mathrm{Mpc}^{-1}$. The Tremaine et al. (2002) estimate of the black hole mass-velocity dispersion relation is

$$
M_{\bullet}=B\left(\sigma / \sigma_{h}\right)^{a},
$$

with $B=1.3 \times 10^{8} m_{\odot}, a=4.0$, and $\sigma_{h}=200$ $\mathrm{km} \mathrm{s}^{-1}$. The product of the two expressions, integrated over $\sigma$, gives the mean mass density,

$$
\rho_{\bullet}=B \phi_{*} \frac{\Gamma((\alpha+a) / \beta)}{\Gamma(\alpha / \beta)}\left(\frac{\sigma_{*}}{\sigma_{h}}\right)^{a} .
$$

The numerical result,

$$
\Omega_{\bullet}(\text { early })=10^{-5.9},
$$

is close to but smaller than the more direct estimate in equation (75). Although the formal uncertainty in equation (79) is smaller it rests on the condition that the Sheth et al. galaxies are a fair sample of the early-type galaxies, which will require careful debate. ${ }^{12}$ Thus in the inventory we quote equation (75).

\subsubsection{Quasar Luminosities and Remnants}

Soltan (1982) and Chokshi \& Turner (1992) have considered the relation between the rate of radiation of energy by quasars and AGNs and the accumulation of mass in the quasar engines, which are assumed to be massive black holes in the centers of galaxies. In this repetition of the calculation we take the number of quasars per unit luminosity and comoving volume to be

$$
L_{*} \frac{d n}{d L}=\frac{L_{*} \Phi\left(L_{*}\right)}{\left(L / L_{*}\right)^{\alpha}+\left(L / L_{*}\right)^{\beta}},
$$

where, from Croom et al. (2004), $\alpha=3.31, \beta=$ 1.09 ,

$$
L_{*} \Phi\left(L_{*}\right)=1.81 \times 10^{-6} \mathrm{Mpc}^{-3},
$$

and the present characteristic luminosity is

$$
L_{*}=6.7 \times 10^{10} L_{B}(\odot) .
$$

In the Croom et al. luminosity evolution model this luminosity evolves as $L_{*}(z) \propto 10^{1.39 z-0.29 z^{2}}$ to redshift $z=2.1$, the deepest redshift used in the Croom et al. analysis. The peak of the observed comoving quasar number density is at $z \simeq 2.5$, and at $2.5<z<5$ the density varies about as $n \propto e^{-1.5 z}$ (Fan et al. 2001, Fig. 3). As a convenient approximation to this behavior we adopt the Croom et al. (2004) luminosity evolution at $z<2.1$, constant comoving luminosity density from $z=2.1$ to $z=3$, and negligibly small luminosity at larger redshifts.

The integral $\int d L L d n / d L$ is the comoving luminosity density, and the time integral multiplied

\footnotetext{
${ }^{12}$ The velocity function of Sheth et al. gives $\left\langle\sigma^{4}\right\rangle^{1 / 4}=180$ $\mathrm{km} \mathrm{s}^{-1}$, compared to our estimate of the characteristic velocity dispersion, $\sigma_{*}=200-220 \mathrm{~km} \mathrm{~s}^{-1}$, in early-type galaxies. Perhaps this is related to the difference.
} 
by the bolometric correction is the net comoving density of energy released. Using the Elvis et al. (1994, Table 17) bolometric correction factor $\mathrm{BC} \equiv L_{\mathrm{bol}} /\left.\left(\nu L_{\nu}\right)\right|_{4450 \AA}=12$, and ignoring the difference between $\mathrm{B}$ and $b_{J}$ passbands (since the quantity that concerns us, $\nu L_{\nu}$ for quasars, is close to flat), we estimate that the integrated energy density released by the quasars is

$$
\Omega_{\mathrm{QSOem}}=8 \times 10^{-8} \text {. }
$$

This uses the solar luminosity,

$$
\nu L_{\nu}(\odot)=2.22 \times 10^{33} \mathrm{erg} \mathrm{s}^{-1},
$$

at $\lambda=4450 \AA$.

Before comparing this estimate to the accumulated mass in black holes let us check consistency with the integrated background radiation. Equation (83) with the Elvis et al. bolometric corrections indicates that the integrated background from quasars at $100 \mu<\lambda<1000 \AA$ is $\Omega \sim 3 \times 10^{-8}$, or $1 \%$ of the total (eqs. [106] plus [107] below). To estimate the expected Xray background we imagine the radiation from the quasars all is emitted at effective redshift $z=2$. In this simple model the present energy density per logarithmic interval of frequency at $2 \mathrm{keV}$ is

$$
\nu \Omega_{\nu}(2 \mathrm{keV})=\frac{\Omega_{\mathrm{QSOem}}}{1+z} \frac{\nu_{e} L_{\nu_{e}}}{\int_{0}^{\infty} L_{\nu} d \nu},
$$

where on the right-hand side $h \nu_{e}=2(1+z) \mathrm{keV}$. The mean spectrum in figure 10 in Elvis et al. (1994) for radio-quiet quasars, with the bolometric factor, indicates that $\nu_{e} L_{\nu_{e}}=\int_{0}^{\infty} L_{\nu} d \nu / 50$ at rest frame energy $h \nu_{e}=6 \mathrm{keV}$. These numbers give the present energy density per logarithmic interval of photon energy $\nu \Omega_{\nu}=10^{-9.3}$ at $2 \mathrm{keV}$. The measured value of the X-ray background is (De Luca \& Molendi 2004) $\nu \Omega_{\nu}=10^{-8.8}$ at $2 \mathrm{keV}$, about three times what is indicated by equations (83) and (85). Since about $80 \%$ of the X-ray background at $2 \mathrm{keV}$ is resolved (Mushotzky et al. 2000; Worsley et al. 2004), these numbers allow room for a significant population of optically faint quasars.

We turn now to the efficiency $\epsilon_{n}$ for production of electromagnetic radiation in the accumulation of the present mass in quasar remnants. If $\epsilon_{n}$ is small the estimate of the integrated mass added to the black holes by the observed energy production by quasars and AGNs is $\Delta \Omega_{\mathrm{BH}}=\Omega_{\mathrm{QSOem}} / \epsilon_{n}$ (eq. [83]). The ratio of this expression to the mass density in massive black holes (the sum of entries 5.6 and 5.7) is our estimate of the radiation efficiency,

$$
\epsilon_{n}=0.02 \text {. }
$$

This is one fifth of the commonly discussed value, $\epsilon_{n} \sim 0.1$. Since our estimate of the X-ray background from optically identified quasars is one third of the measured value, it may be that equation (83) is low by a factor of about three and equation (86) accordingly low by a like factor. A closer check of consistency of the idea that the massive black holes in the centers of galaxies are the quasar remnants awaits advances in surveys of optically faint quasars in broader ranges of wavelength and redshift and better understanding of the quasar emission mechanism. We may also hope that future work will establish the natures of the sources of the harder X-ray background and the possible relevance to the black hole mass budget.

\subsection{Nuclear Binding Energy}

\subsubsection{Heavy Element Abundances}

We consider here the binding energy released by nuclear burning in stars. We normalize the heavy element abundances to the Solar mass fractions in hydrogen, helium, and heavy elements,

$$
X_{\odot}=0.71, \quad Y_{\odot}=0.27, \quad Z_{\odot}=0.019 .
$$

The ratio $Z / X=0.027$ is derived (BP2000) as the initial solar value from $(Z / X)_{\text {solar surface }}=0.0230$ of Grevesse \& Sauval (2000), and $X$ is also the initial value of BP2000. The metallicity in star populations is correlated with the galaxy luminosity. An average over the Schechter luminosity function of the Kobulnicky \& Zaritsky (1999) correlation of the oxygen abundance with the B-band galaxy luminosity, taking $12+\log (\mathrm{O} / \mathrm{H})_{\odot}=8.83$ (Grevesse $\&$ Sauval 2000) $)^{13}$ as the zero point, indicates that

\footnotetext{
${ }^{13}$ Recent work on the solar heavy element abundance suggests a significantly lower oxygen abundance, $12+$ $\log (\mathrm{O} / \mathrm{H})_{\odot}=8.69$ (Allende Prieto, Lambert \& Asplund 2001), but if the heavy element abundances of other elements are scaled down in a similar manner, as indicated by the same team, our net result is not affected. In fact, Bahcall and Pinsonneault (2004) give $(Z / X)_{\text {solar surface }}=$ 0.0176 based on the new abundance, which leaves our result unchanged.
} 
TABLE 3

Heavy Element Masses

\begin{tabular}{|c|c|c|c|c|}
\hline & Objects & Mean Metallicity & Composition & $10^{5} \Omega_{Z}$ \\
\hline 1 & main seq. stars & $\langle Z\rangle$ & solar & 3.2 \\
\hline 2 & substellar objects & $\langle Z\rangle$ & solar & 0.2 \\
\hline 3 & white dwarfs & 1 & $\mathrm{C}+\mathrm{O}$ & 36 \\
\hline 4 & cool gas & $\langle Z\rangle$ & solar & 1.3 \\
\hline 5 & clusters & $Z_{\odot} / 3$ & solar & 1.1 \\
\hline 6 & warm plasma & $Z \odot / 30$ & solar & 2.5 \\
\hline 7 & neutron stars ${ }^{\mathrm{a}}$ & 1 & $(\mathrm{Fe})$ & 5 \\
\hline 8 & stellar mass black holes ${ }^{a}$ & 1 & $(\mathrm{Fe})$ & 6.8 \\
\hline 9 & massive black holes ${ }^{\mathrm{a}}$ & $Z_{\odot}$ & (solar) & 0.005 \\
\hline
\end{tabular}

the mean metallicity in galaxies is

$$
\langle Z\rangle=0.83(1 \pm 0.3) Z_{\odot}=0.016 \pm 0.003 .
$$

Table 3 lists our estimates of the density parameters belonging to the mass in heavy elements in several categories of objects. The entry for main sequence stars in the first line is the product of $\langle Z\rangle$ with the sum of the density parameters in entries 3.3 and 3.4 in the inventory, and the second line uses entry 3.8. Since the main elements in white dwarfs are carbon and oxygen, with thin hydrogen and/or helium layers that typically amount to $\lesssim 0.1 \%$ of the mass, we enter in the third line the density parameter from entry 3.5. We assign the cool gas in entries 3.9 and 3.10 the same mean metallicity as the stars (eq. [88]). The metallicity of the intracluster plasma (entry 3.2) is observed to be about one third of solar (Mushotzky \& Loewenstein 1997; Fukazawa et al. 1998; White 2000), as indicated in Table 3. We suppose the intergalactic plasma (entry 3.1 ) may have metallicty about $3 \%$ of Solar. This likely is larger than the metallicity in plasma in the voids (Penton, Stocke \& Shull 2004), and smaller than the metallicity in the plasma observed as absorption line systems around galaxies (Sembach et al. 2003; Churchill, Vogt, \& Charlton 2003), and perhaps is a reasonable factor-of-three compromise. We assume that neutron stars and stellar mass black hole form by the collapse of an iron core, and that massive black holes grew by the accretion of matter with about the Solar heavy element abundance. These heavy elements are entered in Table 3, but they are now sequestered from the inventory.

Our estimate of the total production of heavy elements, including those that have been lost in neutron stars and black holes, is

$$
\Omega_{Z}=5.7 \pm 1.2 \times 10^{-4} .
$$

The heavy elements in white dwarfs amount to about $65 \%$ of the total. The matter in this large reservoir is liberated only on the rare occasions of Type Ia supernovae.

The model for the rate of Type Ia supernovae is uncertain; we consider the widely accepted Whelan \& Iben (1973) binary white dwarf picture and use a simple model for the supernova rate,

$$
\begin{aligned}
R_{\mathrm{SN} \mathrm{Ia}}(t)= & A \int_{t_{f}}^{t} d t^{\prime} \psi\left(t^{\prime}\right) \int_{m_{\min }}^{8 m \odot} d m \frac{d N}{d m} \times \\
& \exp \left(-\left[t-t^{\prime}-\Delta t\right] / \tau\right),
\end{aligned}
$$

where $A$ is the normalization determined by the empirical Type Ia supernova rate at zero redshift, which is obtained from the three surveys mentioned earlier (eq. [38]),

$$
R_{\mathrm{SNIa}}\left(t_{0}\right)=0.0027_{-0.0008}^{+0.0017}(100 \mathrm{yr})^{-1} \mathrm{Mpc}^{-3},
$$

and $\Delta t(m)=13\left(m / m_{\odot}\right)^{-2.5} \mathrm{Gyr}+\delta$ is the time for the formation of white dwarfs plus the time delay to form the Roche-lobe contact, and we take $\delta=0$ to 1 Gyr. The minimum mass is chosen to be $m_{\min }=\max \left[3 m_{\odot},\left\{\left(t-t^{\prime}\right) / 13 \mathrm{Gyr}\right\}^{-0.4}\right]$, where $3 m_{\odot}$ corresponds to the white dwarf mass $0.7 m_{\odot}$ according to eq. (69) so that the Chandrasekhar limit is met. This is the model taken by Madau et al. (1998) (see also Gal-Yam \& Maoz 2004). We adopt $\tau=4$ Gyr to account for a moderate increase (by a factor of $2 \pm 1$ ) of the observed SNIa occurence to $z=0.6$ (Pain et al. 2002). ${ }^{14}$ The

${ }^{14} \mathrm{~A}$ short time scale is in conflict with the Type Ia super- 
effective time span for the cumulative occurrence of Type Ia supernovae normalised to the rate at $z=0$ is

$$
\begin{aligned}
T_{\mathrm{eff}} & =R_{\mathrm{SNIa}}\left(t_{0}\right)^{-1} \int_{t_{f}}^{t_{0}} R_{\mathrm{SNIa}}(t) d t \\
& =15 \pm 3 \mathrm{Gyr},
\end{aligned}
$$

for our model of star formation (eqs. [31] and [33] with $z_{f}=2.5$ to 4 ). The cumulative comoving number density of supernova in this model is $4.0 \times$ $10^{5} \mathrm{Mpc}^{-3}$. Since we estimate that the number density of white dwarfs created is $8 \times 10^{7} \mathrm{Mpc}^{-3}$, this would mean that about $1 \%$ of the white dwarfs have been disrupted. If $0.7 m_{\odot}$ of ${ }^{56} \mathrm{Ni}$ is produced in each supernova (e.g., Branch 1992), the mass density of iron-group elements produced by type Ia supernovae is $\Omega_{\mathrm{Fe}}=2.0 \times 10^{-6}$. This is supplemented with $\Omega_{\mathrm{Fe}}=3.6 \times 10^{-6}$ from Type II $+\mathrm{Ib} / \mathrm{c}$ supernovae (see eq. [39]), which produces $0.075 \mathrm{~m}_{\odot}$ of iron per event (Arnett 1996; Weaver, Zimmerman \& Woosley 1978). These estimates indicate that the total density parameter in the iron group elements is

$$
\Omega_{\mathrm{Fe}}=6 \times 10^{-6},
$$

of which about $60 \%$ is from Type II supernovae.

This estimate can be compared to the product of the mass in heavy elements not locked up in stellar remnants (the sum of lines 1, 2, and 4 to 6 in Table 3),

$$
\Omega_{Z}^{\prime}=0.8 \pm 0.25 \times 10^{-4} .
$$

with the iron group mass fraction (Grevesse \& Sauval 2000),

$$
\Omega_{\mathrm{Fe}}=0.077 \Omega_{Z}^{\prime}=6.3 \times 10^{-6} .
$$

The two approaches give a consistent picture for the origin of iron and the supernova rates.

nova rates in early type galaxies. With $\tau=4 \mathrm{Gyr}$, the SN fraction in early type galaxies is 0.30 at $z \approx 0$, which is consistent with the observed value, $0.35_{-0.10}^{+0.15}$. Here we have identified stars formed at $z>0.7$ as an early population that is partitioned into early-type galaxies and bulges of disk galaxies according to the FHP fractions of bulge luminosities. The fraction drops to $<0.25$ if $\tau=3 \mathrm{Gyr}$. The observed rates of Type Ia supernovae in morphologically separated galaxies seem to be proportional to the $r$ band luminosity density; the luminosity density in E/S0 galaxies is $31 \%$ of the total (Nakamura et al. 2003). This would suggest a close to constant supernova rate.
The growth of the abundance of heavy elements is accompanied by the accumulation of helium, apart from the heavy elements that enter white dwarfs, neutron stars and stellar mass black holes. The estimate of $\Delta Y / \Delta Z$ in equation (20), applied to all entries in Table 3 except 3,7 and 8 , is our estimate of the present helium mass fraction in excess of primeval,

$$
\Delta \Omega_{Y}=\frac{\Delta Y}{\Delta Z} \Omega_{Z}^{\prime}=1.7 \pm 1.0 \times 10^{-4} .
$$

This is consistent with our estimate from the products of stellar evolution (eq. [120]).

There are in the literature many analyses of the stellar production of heavy elements (see for example Calura \& Matteucci 2004 and references therein). Our results are generally consistent for relevant entries. Important differences are our inclusion of heavy elements in white dwarfs, which dominate the entry, and our consideration of iron in the progenitors of supernovae. These components are crucial to the consideration of the balance between the nuclear binding energy stored in the elements and the energy released in radiation.

\subsubsection{Nuclear Binding Energies}

Our definition of the nuclear binding energies of the heavy elements differs from the usual practice in tables of nuclei. Because we are interested in the release of energy in the formation of the heavy elements, we calculate the binding energy with respect to free protons and electrons.

We write the energy released in the production of the heavy elements present in the interstellar medium, and in stars when they formed, as

$$
\Omega_{\mathrm{NB}, \mathrm{Z}}=(0.0081+0.0071 \Delta Y / \Delta Z) \Omega_{Z},
$$

where 0.0081 is the energy generation efficiency factor for the solar composition. The nuclear binding energy in substellar objects and in diffuse matter, in entries $6.2,6.4$, and 6.5 in Table 1 , is computed from this equation with the heavy element masses in Table 3. For main sequence stars, we add to equation (97) the nuclear binding energy associated with the helium that has been produced in the stars, which we take to be on average $5 \%$ of the star mass, that is, half the helium a star produces while it is on the main sequence. The sum is our estimate of the nuclear binding energy in stars in entry 6.1. 
Entry 6.3 , for white dwarfs, is

$$
\begin{aligned}
\Omega_{\mathrm{NB}, \mathrm{wd}} & =\left[0.0080\left(1-Y_{p}\right)+0.0010 Y_{p}\right] \Omega_{Z} \\
& =10^{-5.6} .
\end{aligned}
$$

The second term in parentheses accounts for the primordial helium abundance (eq. [18]). The value of $\Omega_{Z}$ in the first line is taken from line 3 of Table 3 . This component amounts to about $40 \%$ of the nuclear binding energy. The large amount of nuclear burning in the stellar giant and supergiant phases is discussed in connection with Table 5 below.

The nuclear binding energy in the matter out of which a neutron star formed was converted to gravitational binding energy by the dissociation of the heavy elements during a supernova. The nuclear binding energy released in the formation of the heavy elements that were part of the raw material for a neutron star added to the radiation background, of course, but that accounting now belongs in category 5 . The total entered in the inventory for category 6 accordingly is about $15 \%$ smaller than the sum of the nuclear binding energies in all entries in Table 3. The nuclear binding energy to compare to the energy required to produce the radiation background is the full sum, including iron core progenitors for neutron stars and black holes,

$$
\Omega_{\mathrm{NB}}=-5.7 \pm 1.3 \times 10^{-6} .
$$

We discuss the relation to the energy density in radiation in the next subsection.

\subsection{The Radiation Backgrounds}

The cosmic energy density in electromagnetic radiation is thought to be dominated by mildly redshifted starlight, at $\lambda \sim 1 \mu$, and a far infrared peak at $\lambda \sim 100 \mu$ that is produced by the absorption and reradiation of starlight and the light from AGNs (Hauser \& Dwek 2001 and references therein). The energy densities at radio and X-ray to $\gamma$-ray wavelengths are much smaller, but they are useful measures of high energy processes, as is the gravitational wave background. Neutrino production might be counted as part of the radiation backgrounds, but we find it convenient to enter neutrinos in a separate category.

\subsubsection{The $\lambda \sim 1 \mu$ Background}

Observations of the optical to near infrared extragalactic background light that report positive detections are summarized in Table 4. They suggest that the surface brightness per logarithmic interval in frequency is about constant at $\nu I_{\nu}=20 \pm 5 \mathrm{nW} \mathrm{m}^{-2} \mathrm{sr}^{-1}$ in the range $3500 \AA$ to $3.5 \mu$. This corresponds to energy density

$$
\Omega_{\mathrm{opt}}=2.3 \pm 0.6 \times 10^{-6},
$$

in the optical to near infrared. In view of the technical difficulty of these observations, equation (100) may conservatively be taken as an upper limit. Integrated galaxy number counts give surface brightness typically one third of the entries in Table 4 (Madau \& Pozzetti 2000; see also Hauser \& Dwek 2001, Table 3),

$$
\Omega_{\mathrm{opt}}=0.9 \pm 0.2 \times 10^{-6} .
$$

This might be considered a lower limit.

We have a check from the energy density computed as a time integral of the luminosity density,

$$
u=\int \frac{d \lambda}{\lambda} \int_{0}^{t_{o}} \frac{d t}{1+z} \lambda(t) \mathcal{L}_{\lambda(t)} .
$$

The integral is from high redshift to the present world time, $t_{o}$. The integrand is the luminosity per logarithmic interval of wavelength and comoving volume, corrected by the redshift factor, $1+z=$ $1 / a(t)$, and evaluated at the redshifted wavelength $\lambda(t)=a(t) \lambda_{o}$, where the observed wavelength is $\lambda_{o}$ and the expansion parameter $a(t)$ is normalized to unity at the present epoch. In the flat cosmological model the integral is

$$
\begin{aligned}
& u=H_{o}^{-1} \lambda_{o} \mathcal{L}_{\lambda_{o}} I, \\
& I=\int_{0}^{1} \frac{d a}{\sqrt{\Omega_{m} / a^{3}+1-\Omega_{\lambda}}} \int \frac{d \lambda \mathcal{L}(\lambda, z)}{\lambda_{o} \mathcal{L}_{\lambda_{o}}}(103)
\end{aligned}
$$

We take the shape of the present cosmic spectrum $\lambda \mathcal{L}_{\lambda}$ as a function of wavelength from Figure 13 in Blanton et al. (2003), and we normalize to the luminosity density at $\lambda \sim 1 \mu$ in equation (14). We approximate the evolution of the comoving luminosity density by extrapolating the Rudnick et al. (2003) power law fits to the evolution in the rest frame U, V and B bands, in the form

$$
\mathcal{L}(\lambda, z) \propto(1+z)^{\beta}, \beta=0.93(4400 \AA / \lambda)^{2.5} .
$$


TABLE 4

RADIATION BACKGROUND

\begin{tabular}{cc}
\hline \hline$\lambda(\mu)$ & $\nu I_{\nu}\left(\mathrm{nW} \mathrm{m}^{-2} \mathrm{sr}^{-1}\right)$ \\
\hline 0.10 & $0.60^{\mathrm{a}}$ \\
0.30 & $13 \pm 8^{\mathrm{b}}$ \\
0.37 & $24 \pm 8^{\mathrm{c}}$ \\
0.55 & $18 \pm 8^{\mathrm{b}}$ \\
0.81 & $24 \pm 9^{\mathrm{b}}$ \\
2.2 & $23 \pm 9^{\mathrm{d}}$ \\
3.5 & $12 \pm 3^{\mathrm{d}}$ \\
\hline & \\
${ }^{\mathrm{a}}$ Henry (1999) & ${ }^{\mathrm{b}}$ Bernstein et al. (2002) \\
${ }^{\mathrm{c}}$ Matilla (1990) & ${ }^{\mathrm{d}}$ Wright \& Reese (2000)
\end{tabular}

We truncate the integral at $z=3$, the limit of the Rudnick et al. measurements. In this model the dimensionless integral in equation (103) is $I=$ 1.35. (If the comoving luminosity density were constant the integral would be $I=0.82$.) The result is

$$
\Omega_{\mathrm{opt}}=1.5 \times 10^{-6} .
$$

For the inventory we adopt

$$
\Omega_{\mathrm{opt}}=1.6 \pm 0.7 \times 10^{-6} .
$$

The error spans the estimates based on measurements of the surface brightness of the sky (eqs. [100]), the source counts (eq. [101]) and the luminosity density (eq. [105]).

\subsubsection{The Far Infrared Background}

The COBE DIRBE (Hauser et al. 1998) and FIRAS (Fixsen et al. 1998) experiments detect the extragalactic radiation background at $\lambda \gtrsim 100 \mu$. The integral for $\lambda>125 \mu$ is $u=14 \mathrm{nW} \mathrm{m}^{-2} \mathrm{sr}^{-1}$ (Fixsen et al. 1998). Extending the integration to $100 \mu$ might reasonably be expected to add 2 $\mathrm{nW} \mathrm{m} \mathrm{m}^{-2} \mathrm{sr}^{-1}$ to this value. The density parameter for the sum is

$$
\Omega_{\mathrm{FIR}}=0.8 \pm 0.2 \times 10^{-6} .
$$

This is entry 7.2 in the inventory. It seems to be a believable lower bound on the energy density in the far infrared. The radiation measurements allow room for a comparable amount of energy at $30 \lesssim \lambda \lesssim 100 \mu$ (Finkbeiner, Davis \& Schlegel 2000; see also Hauser \& Dwek 2001). However, this amount of energy shortward of $100 \mu$ wavelength would distort the $\mathrm{TeV} \gamma$-ray spectrum from the extragalactic source Mrk501 (Quinn 1996; Aharonian et al. 1999), by the absorption due to $e^{+} e^{-}$pair production (Kneiske, Mannheim \& Hartmann 2002; Konopelko et al. 2003). Thus it appears that equation (107) is close to the total in the far infrared.

The optical and infrared light from quasars is about one percent of the starlight and far infrared backgrounds ( $\$ 2.5 .4)$.

\subsubsection{A Check: Nuclear Burning}

We may compare the estimate of the present energy density in the optical through the far infrared against what would be expected from the energy stored in the heavy elements (eq. [99]), and what would be expected from the picture for stellar evolution. We comment on the former here and the latter in $§ 2.8 .1$.

The sum of equations (106) and (107) (entries 7.2 and 7.3), corrected for the redshift energy loss factor in equation (35), is an estimate of the nuclear energy required to produce the observed radiation,

$$
\Omega_{\text {photon }}=4.8 \pm 1.4 \times 10^{-6} .
$$

This number may be compared to the nuclear binding energy released by nucleosynthesis. The estimate in equation (99), reduced by $7 \%$ to take account of the energy carried away by neutrinos, is

$$
\Omega_{\text {photon, nuc. }}=5.3 \pm 1.1 \times 10^{-6} \text {. }
$$

The difference, $10 \%$, is well within our uncertainties. It might be relevant to note that the difference between equations (108) and (109) would be 
increased if we adopted the estimate of the optical background from source counts.

\subsubsection{The X-ray - $\gamma$-Ray Background}

Entry 7.4 in Table 1 is the integral of the radiation background spectrum compiled by Gruber et al. (1999) over the energy range $3 \mathrm{keV}$ to $100 \mathrm{GeV}$. The largest contribution to the integral is at photon energy $\sim 30 \mathrm{keV}$, but the convergence at high energy is slow because the energy per logarithmic interval of frequency varies about as $\nu \Omega_{\nu} \sim \nu^{-0.1}$. The measurement at $3 \mathrm{keV}$ by De Luca \& Molendi (2004) is about 1.3 times the Gruber et al. value. We have not attempted to adjust the entry for this more recent result because we do not know its significance for the spectrum at higher energies.

\subsubsection{The Radio Background}

Longair (1995), following Bridle's (1967) study, concludes that the brightness temperature of the isotropic radio background at $178 \mathrm{MHz}$, after correction for the thermal component, is $T=27 \pm 7 \mathrm{~K}$. This corresponds to

$$
u_{\nu}=6600(1 \pm 0.25)\left(\frac{\nu}{1 \mathrm{GHz}}\right)^{-0.8} \mathrm{Jy} \mathrm{sr}^{-1} \text {, }
$$

assuming the canonical power law spectrum (see also Peacock 1995). Longair also argues that this radiation is dominated by galaxies.

Equation (110) may be compared to the sum of the radio source counts in the $9 \mathrm{CR}$ radio survey at $15 \mathrm{GHz}$, in the observed range of flux densities, 0.01 to $1 \mathrm{Jy}$ (Waldram et al. 2003). This sum gives $u_{\nu}=5900 \mathrm{Jy} \mathrm{sr}^{-1}$ when scaled to $1 \mathrm{GHz}$ with the power index of 0.8 . The radio source counts at $8.4 \mathrm{GHz}$ by Fomalont et al. (2002), summed over $10 \mu \mathrm{Jy}$ to $1 \mathrm{Jy}$, give $u_{\nu}=4700 \mathrm{Jy} \mathrm{sr}^{-1}$ at $1 \mathrm{GHz}$. A comparable result is obtained from the count at 40 GHZ (as summarized in Figure 13 of Bennett et al. 2003b). Haarsma \& Partridge (1998) argue that the integral over the counts likely converges at $S \sim 1 \mu \mathrm{Jy}$. We conclude that the measurements are reasonably concordant with equation (110), within $\sim 0.2$ dex.

The integral of equation (110) slowly diverges at short wavelengths. We adopt, as an operational definition, a cutoff at $\lambda=1 \mathrm{~mm}(\nu=300 \mathrm{GHz})$, and we count the contribution at shorter wavelengths as part of the FIR background in our in- ventory. There is a natural cutoff at long wavelength, at $\nu \sim 3 \mathrm{MHz}$ (Simon 1978). Integrating eq. (110) over this wavelength range, we obtain

$$
\Omega_{\text {radio }}=5 \times 10^{-11} \text {. }
$$

To understand the relation to other energy entries, we may attempt an alternative estimate by directly summing the contributions of known radio galaxies. The luminosity function of radio galaxies at zero redshift is now reasonably well known by virtue of the correlation of a large NRAO VLA Sky Survey at $1.4 \mathrm{GHz}$ (NVSS; Condon et al. 1998) with galaxies in optical catalogues (Condon, Cotton \& Broderick 2002 [UGC vs. NVSS]; Machalski \& Godlowski 2000 [LCRS vs. NVSS]; Sadler et al. 2002 [2dF vs. NVSS]). The luminosity function is written as the sum of two components, weak radio emitters that represent normal galaxies with star forming activity, and strong emitters that mostly consist of subsets of giant elliptical galaxies and AGNs. The former activity is ascribed to electron acceleration in Type II (and Ib/c) supernova remnants. At close to zero redshift the integral over the luminosity function gives luminosity densities

$$
\begin{aligned}
\mathcal{L}_{g} & =1.5 \times 10^{19} \mathrm{~W} \mathrm{~Hz}^{-1} \mathrm{Mpc}^{-3} \\
\mathcal{L}_{a} & =3.7 \times 10^{19} \mathrm{~W} \mathrm{~Hz}^{-1} \mathrm{Mpc}^{-3}
\end{aligned}
$$

at $1.4 \mathrm{GHz}$ (Condon et al. 2002). The second component, AGNs, is dominated by the most luminous sources, and so is subject to sampling fluctuations. Haarsma et al. (2000) show that the evolution of the first component in equation (112) is fast to $z \sim 1$. The evolution they derive is consistent with the model for the evolution of the star formation rate in equation (31). Condon (1992) gives the relation between the radio emissivity and the star formation rate. After adjustment for the overall constraint from the star formation rate density derived from the local $\mathrm{H} \alpha$ luminosity density, as discussed in $\S 2.3 .2$, and our reference IMF, the relation reads

$$
\begin{aligned}
u_{\nu}= & 2.5 \times 10^{21}\left(\frac{\nu}{\mathrm{GHz}}\right)^{-0.8} \\
& +2.6 \times 10^{20}\left(\frac{\nu}{\mathrm{GHz}}\right)^{-0.1} \mathrm{~W} \mathrm{~Hz}^{-1},
\end{aligned}
$$

per $1 m_{\odot} \mathrm{yr}^{-1}$ of star formation. The first component represents synchrotron radiation, primarily from supernovae, and the second represents bremsstrahlung from HII regions. The 
bremsstrahlung contribution becomes more important above $25 \mathrm{GHz}$. The integral over the star formation history to $z_{f}=3$ (with the redshift energy loss) and the frequency range $\nu=3 \mathrm{MHz}$ to $300 \mathrm{GHz}$ gives $\Omega_{\text {radio }}=3.3 \times 10^{-11}$, where the contributions from synchrotron radiation and bremsstrahlung are in the proportion 0.4:0.6 in the frequency range that concerns us.

The evolution of the strong radio sources appears not to be very fast. The luminosity functions of Sadler et al. (2002) and Machalski \& Godlowski (2000) up to $z \sim 0.2$ suggest a slow evolution compared with that of the weak emitter component, at least at low redshifts. We take the evolution factor derived from high redshift strong radio galaxies by Willott et al. (2001), $(1+z)^{2.6} E(z)$ with $E(z)=\left[\Omega_{m}(1+z)^{3}+\Omega_{\lambda}\right]^{1 / 2}$, and we assume the canonical synchrotron spectrum. The integral over time and frequency yields $\Omega_{\text {radio }}=2.0 \times 10^{-11}$. The sum of the two components is

$$
\Omega_{\text {radio }}=5 \times 10^{-11} .
$$

The consistency with equation (111) suggests that this simple model for the radio sources is a useful approximation.

\subsubsection{Gravitational Radiation}

We consider two sources of gravitational radiation, the merging of stellar mass binaries and the formation of massive black holes in the centers of galaxies. The rate of production of gravitational radiation by stellar mass binaries in the Milky Way depends on the very uncertain distribution of properties of close binary systems that contain white dwarfs, neutron stars, and black holes. The largest contribution to the mean gravitational radiation luminosity of the Milky Way is thought to be from the merging of binary neutron stars, as indicated in Fig. 12 in Schneider et al. (2001).

Shibata \& Uryū (2002) and Faber et al. (2002) find that in the merging of a binary neutron star system the energy emitted as gravitational radiation is

$$
\epsilon_{\mathrm{GWnn}} \simeq 0.005
$$

times the mass of the binary. A major uncertainty is the behavior of the system after contact. Gravitational radiation emitted during the transient formation of an oscillating neutron star might double the number in equation (115).
If we adopt the estimate of the rate of merging of binary neutron stars in the Milky Way, $\mathcal{R} \approx 80 \mathrm{Myr}^{-1}$, that is based on the three known systems that will merge within the Hubble time (Kalogera et al. 2004a, b), then we find that the product with the radiation energy released (eq. [115]), the effective number density of Milky Way galaxies (eq. [54]), and 85 Gyr/2 (eqs. [35] and [36]) is the gravitational radiation energy density $\Omega_{\mathrm{NS}, \mathrm{gw}}=10^{-9}$. The merger rate is higly uncertain: the spread of central values in the models in Table 1 in Kalogera et al. (2004b) is $10_{-1}^{+0.7}$. On taking account of the upward uncertainty in equation (115), we are led to allocate an error of a full order of magnitude up or down in entry 7.5.

The merging rates for neutron star-black hole and black hole-black hole binaries are even more uncertain. The indication from the analysis of Schneider et al. (2001) is that mergers involving black holes add about $10 \%$ to the gravitational radiation luminosity.

In the low frequency regime $(\sim \mathrm{mHz})$ the luminosity is dominated by compact white dwarf binaries, as shown by Hils, Bender \& Webbink (1990). The integration of the gravitational lumiosity given in their Table 7 with the number of close white dwarf binaries in the Milky Way normalised to $3 \times 10^{6}$ (after a suggested reduction by a factor of ten from the simple theoretical estimate of the abundance of these objects) gives the luminosity $3 \times 10^{39} \mathrm{erg} \mathrm{s}^{-1}$. The product with the effective number density of Milky Way galaxies and the effective time span with redshift correction gives $\Omega_{\mathrm{WD}, \mathrm{gw}} \sim 10^{-10}$. This is at the lower end of the suggested range of uncertainty in the value of $\Omega_{\mathrm{NS}, \mathrm{gw}}$.

Our estimate of the gravitational radiation released by the formation of the massive black holes in the centers of galaxies is based on the analysis by Baker et al. (2001), which indicates that the gravitational radiation energy released by the merging of equal mass black holes is $\epsilon_{\text {ngw }} \simeq 0.03$ times the final black hole mass $M$, and that the frequency of the radiation is on the order of $0.1 M^{-1}$, or about $10^{-4} M_{9} \mathrm{~Hz}$ when the mass is measured in units of $10^{9} \mathrm{~m}_{\odot}$. We take the redshift at peak production to be $z_{n} \sim 2.5$, about the peak of quasar activity. Then the present energy density of the gravitational radiation from the formation of the massive black holes, with mass density 
$\Omega_{n}=10^{-5.4}$, is $\Omega_{\mathrm{ngw}} \sim \epsilon_{\mathrm{ngw}} \Omega_{n} /\left(1+z_{n}\right) \sim 10^{-7.5}$. This may be compared to the bound on the gravitational wave energy density from pulsar timing measurements, $\Omega_{g}<10^{-8}$ at frequency $\sim 10^{-8} \mathrm{~Hz}$ (Kaspi, Taylor \& Ryba 1994; Thorsett \& Dewey 1996; Lommen \& Backer 2001). The bound is about $30 \%$ of our estimate of $\Omega_{\mathrm{ngw}}$, but there is no inconsistency because the expected peak frequency is considerably larger than the frequency where the pulsar constraint is tight. The spectrum of the gravitational radiation from merging massive black holes, and the puzzle of how merging black holes get close enough that energy loss by gravitational radiation drives merging within a Hubble time, are discussed by Hughes et al. (2001), Jaffe \& Backer (2003) and Wyithe \& Loeb (2003).

An entry in the inventory for possible sources of low frequency gravitational radiation in the early universe, as from inflation or phase transitions, seems premature.

\subsection{Products of Stellar Evolution}

It is in principle straightforward to compute the integrated outputs of stellar evolution - energy, neutrinos, helium, and heavy elements - given models for the IMF, the star formation history, and stellar evolution. Since the details of the results of stellar evolution computations are not easily assembled, we use approximate estimates by procedures similar to those developed in $\S 2.3 .1$ and 2.3.2 for the stellar population and its evolution. The results add to the checks of consistency of our estimates of the stellar production of helium and heavy elements and the resulting total energy release, and are used to estimate the inventory entries for the neutrino cosmic energy density.

\subsubsection{Stellar Evolution}

Most stars with masses $m<1 m_{\odot}$ are still on the main sequence. We assume that on average $5 \%$ of the hydrogen in these subsolar stars has been consumed, with energy production efficiency 0.0071. Most of the stars with masses $m>1 m_{\odot}$ have already undergone full evolution and left compact remnants, while the fraction $\sim\left(m / m_{\odot}\right)^{-2.5}$ is still on the main sequence. We assume that in the latter stars on average $5 \%$ of the hydrogen has been consumed, as for subsolar stars. For the evolved stars we do not attempt to follow the details of nuclear burning and mass loss. Instead, we adopt estimates of the nuclear fuel consumed or mass lost in a few discrete stages of evolution, in a similar fashion to the approach used in $§ 2.3 .1$ to tally stellar remnants.

When the amount of hydrogen consumed in stellar burning is $10 \%$ of the mass of a star it leaves the main sequence. In the model in $\S 2.3 .1$, stars with masses in the range $1<m<8 m_{\odot}$ eventually produce white dwarfs that mainly consist of a carbon-oxygen core. In standard stellar evolution models, hydrogen burning extends outward to a shell after core hydrogen exhaustion, and helium burning similarly continues in a shell after core helium exhaustion. That leaves a CO core with the mass given by equation (69). The helium layer outside the $\mathrm{CO}$ core is thin for $m<2.2 m_{\odot}$, for which helium ignition takes place as a flash, but in stars with masses $m>2.2 m_{\odot}$ a significant amount of helium is produced outside the core, transported by convection to the envelope, and liberated. A $5 m_{\odot}$ star liberates $0.4 m_{\odot}$ of helium and produces a core with mass $0.85 m_{\odot}$ (e.g., Kippenhahn, Thomas \& Weigert 1965). We model the helium production as a function of the initial star mass $m$ as

$$
m_{\mathrm{He}}=0.23(m-2.2)+0.63,
$$

in solar mass units. The energy production in hydrogen shell burning is the product of this mass with the post-stellar hydrogen mass fraction, 0.71 (eq. [87]), and the efficiency factor, 0.0071. Helium burning in the core produces energy with efficiency factor 0.0010 .

For stars with masses $m>8 m_{\odot}$ we adopt the helium yield from Table 14.6 of Arnett (1996), ${ }^{15}$ which we parametrise as

$$
m_{\mathrm{He}}=0.69 m-3.9 .
$$

This connects to equation (116) at $8.7 m_{\odot}$. We take the $\mathrm{CO}$ core mass as a function of the initial stellar mass from Arnett (1996):

$$
m_{\mathrm{CO}}=0.28 m-2.20, \quad m>13 m_{\odot} .
$$

We use an interpolation of eqs. [69] and [118] for stellar masses between 8 and $13 m_{\odot}$. The energy

${ }^{15}$ We prefer eq.[117] which is derived from tabulated values, rather than Arnett's parametrisation, $m_{\mathrm{He}}=0.43 m-2.46$. 
TABLE 5

Stellar ENERGy PRODUCTION ${ }^{\mathrm{a}}$

\begin{tabular}{lcccc}
\hline \hline stage of stellar evolution & $0.08-1 m_{\odot}$ & $1-8 m_{\odot}$ & $8-100 m_{\odot}$ & sum \\
\hline main sequence & 0.11 & 0.20 & 0.12 & 0.43 \\
H shell burning & & 0.18 & 0.29 & 0.48 \\
core evolution & & 0.05 & 0.04 & 0.09 \\
\hline sum & 0.11 & 0.43 & 0.46 & 1.00 \\
\hline
\end{tabular}

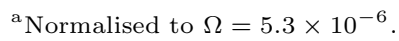

release is $0.0071 \times 0.71$ per unit mass for He production, and 0.0014 for CO core formation and the further heavy element production.

The energy output obtained by integration over the IMF and PDMF is

$$
\Omega_{\text {stellar E }}=5.3 \times 10^{-6} .
$$

The partition into each phase of stellar evolution and stellar mass range is given in Table 5, where the numbers are normalised to equation (119). About $60 \%$ of the energy is produced in the evolved stages.

The estimate of the total energy generation in equation (119) is in satisfactory agreement with our estimate of the nuclear binding energy, $\Omega_{\mathrm{BE}}=$ $5.7 \times 10^{-6}$ (eq. [99]), and the energy production required to produce our estimate of the present radiation energy density, $\Omega_{\gamma+\nu}=(5.1 \pm 1.5) \times$ $10^{-6}$ (eq. [108] corrected for neutrino emission, as discussed in $\S 2.8 .2$ ). That is, our models for stellar formation and evolution are consistent with our estimates of the accumulation of heavy elements and the stellar background radiation.

In our models for stellar formation and evolution the mass of helium that has been liberated to interstellar space is

$$
\Delta \Omega_{Y} \approx 2.1 \times 10^{-4}
$$

This may be compared to our estimate from the production of heavy elements and the associated production of helium, $\Delta \Omega_{Y}=1.7 \pm 1 \times 10^{-4}$ (eq. [96]). The heavy element production is calculated in a similar way. The current wisdom is that a neutron star or black hole is left at the end of the evolution of a star with mass $m>8 m_{\odot}$, and the rest of the mass is returned to the interstellar medium. On subtracting the remnant mass (indicated in Table 2) from the total heavy element mass produced, we find that in our model the heavy element production is

$$
\Omega_{Z} \approx 5 \times 10^{-5}
$$

The more direct estimate in equation (94) is $\Omega_{Z}^{\prime} \simeq$ $8 \times 10^{-5}$.

We suspect the checks on helium and heavy element production in equations (120) and (121) are as successful as could be expected. In particular, equation (118) for the CO core mass is not tightly constrained. One must consider also the possibility that some supernovae do not produce compact remnants, as is suggested by the cases of Cas A and SN1987A. The maximum heavy element production when there are no remnant neutron stars or black holes is three times the value in equation (121), and larger than $\Omega_{Z}^{\prime}$ (eq. [94]).

\subsubsection{Neutrinos from Stellar Evolution}

We need the fraction $f_{\nu}$ of the energy released as neutrinos by the various processes of nuclear burning. In stars with masses $m<1.4 m_{\odot}$, energy generation in hydrogen burning is dominated by the slow reaction $p+p \rightarrow d+e^{+}+\nu_{e}$. This produces neutrinos with mean energy $0.265 \mathrm{MeV}$, which amounts to $f_{\nu}=0.020$ times the energy generated in helium synthesis. In a Solar mass star electron capture of ${ }^{7} \mathrm{Be}$ adds 0.005 to the fraction $f_{\nu}$. The neutrino energy emission fraction is larger in higher mass $\left(m>1.4 m_{\odot}\right)$ main-sequence stars in which the $\mathrm{CNO}$ cycle dominates. In this process, the neutrinos produced in the $\beta^{+}$decays of ${ }^{13} \mathrm{~N}$ and ${ }^{15} \mathrm{O}$ carry away the energy fraction $f_{\nu}=0.064$. The fraction increases to 0.075 for $m \gtrsim 2 m_{\odot}$, when the subchain ${ }^{15} \mathrm{~N}-{ }^{16} \mathrm{O}-{ }^{17} \mathrm{~F}-{ }^{17} \mathrm{O}-$ ${ }^{14} \mathrm{~N}$ starts dominating and neutrinos are produced 
by the beta decay of ${ }^{17} \mathrm{~F}$. This sidechain also dominates during shell burning.

The integral of these factors over the stellar IMF normalised to the present-day mass density $\Omega_{\text {star }}=0.0027$ (eq. [27]), together with our prescription for energy generation in Table 5, yields the neutrino energy production,

$$
\Omega_{\nu, \mathrm{ms}}=0.31 \times 10^{-6} .
$$

The temperatures and densities that are reached up to carbon burning are low enough that there is negligible neutrino energy loss from neutrino pair production processes.

The temperatures after carbon burning are high enough that the neutrino energy loss dominates, that is, $f_{\nu} \simeq 1$ (Weaver et al. 1978). Thus we may take it that the extra binding energy $\Delta(\mathrm{BE})$ of the elements heavier than ${ }^{20} \mathrm{Ne}$ with respect to the binding energy of carbon represents the neutrino energy emitted in the late stages of stellar evolution. On multiplying the heavy element mass (the sum of entries 1,2 and 4 to 9 in Table 3 ) by $\sum\left(Z_{i} / Z\right) \Delta(\mathrm{BE})_{i}$ (where $\langle\Delta(\mathrm{BE})\rangle=0.0004$ for the solar mix of element abundances, and $\sum Z_{i} / Z=0.35$ is from Grevesse \& Sauval (2000), we obtain

$$
\Omega_{\nu, \text { pairs }}=0.03 \times 10^{-6} .
$$

The sum of equations (122) and (123) is $7 \%$ of the total energy production (eq. [119]). The present energy density of the stellar neutrinos, in entry 8.1 in Table 1 , is the product of this sum with the redshift loss factor $\sim 0.5$ (eq. [35]).

\subsubsection{White Dwarfs and Neutron Stars}

Most of the gravitational energy liberated in white dwarf formation goes to neutrinos, and in the latest stage to X-rays. Since the latter is small the contribution to the neutrino energy density (entry 8.2 in the inventory) is the product of the gravitational binding energy in entry 5.3 with the redshift factor.

More than $99 \%$ of the energy released in core collapse also is carried away by neutrinos. Thus we similarly obtain entry 8.3 by multiplying entry 5.4 by the redshift factor.

\subsection{Cosmic Rays and Magnetic Fields}

This estimate is based on the rate of production of cosmic rays in the Milky Way galaxy. We start from the radio structure. In their analysis of the Haslam et al. (1982) measurements, Beuermann, Kanbach, \& Berkhuijsen (1985) write the radio luminosity distribution at $408 \mathrm{MHz}$ as the sum of thin and thick components. We model each distribution, in an approximation to their results, as

$$
\left.\mathcal{E}(\vec{r})=\mathcal{E}(0) e^{-\left(|z| / z_{\mathrm{eq}}+r / l\right.}\right) .
$$

In the thick component the scale heights in the disk and perpendicular to the disk are

$$
l=3.1 \mathrm{kpc}, \quad z_{\mathrm{eq}}=1.4 \mathrm{kpc},
$$

scaled to Solar galactocentric distance $8 \mathrm{kpc}$ (see Appendix). The total luminosity in the thin component in this model may be neglected, but the thin component is a significant contribution to the local luminosity density, $\mathcal{E}_{\odot}$ : the local thick component fraction is $\mathcal{E}_{\odot}($ thick $) / \mathcal{E}_{\odot}=0.55$. We assume the energy density in cosmic rays is proportional to the luminosity density at $408 \mathrm{MHz}$. A more detailed model would take account of the spatial variation of the magnetic field strength, but that will be left for future studies. We normalize to the local energy density, $1.8 \mathrm{eV} \mathrm{cm}^{-3}$, in relativistic cosmic rays (Webber 1998). In these approximations the energy of cosmic rays in the galaxy is $10^{56.1} \mathrm{erg}$. We take the cosmic ray mean life in the galaxy to be $2 \times 10^{7} \mathrm{yr}$ (Garcia-Munoz, Mason \& Simpson 1977; Yanasak et al. 2001). The ratio is a measure of the cosmic ray luminosity, $10^{41.3} \mathrm{erg} \mathrm{s}^{-1}$. If the acceleration of cosmic rays is mainly due to shocks of Type II and Ibc supernovae, ${ }^{16}$ following the conventional wisdom (Ginzburg \& Syrovatskii 1964), the cosmic ray luminosity is proportional to the star formation rate. Thus the product of this luminosity with the effective time (eq. 36) and the redshift factor (eq. [35]) is an estimate of the contribution of the Milky Way to the present energy in cosmic rays. The product with the effective number density of Milky Way galaxies (eq. [54]) is our estimate of the present

\footnotetext{
${ }^{16}$ No type Ia supernova remnants are known to give strong radio sources (Weiler et al. 1986). This implies that Type Ia supernovae contribute little to the acceleration of cosmic rays.
} 
energy density in cosmic rays from normal galaxies,

$$
\Omega_{\mathrm{CR}}=10^{-8.3} .
$$

The radio luminosity of the Milky Way offers a check of this calculation. The Beuermann et al. (1985) luminosity of the Milky Way, $5.5 \times 10^{21}$ $\mathrm{W} \mathrm{Hz}{ }^{-1}$ at $\nu=408 \mathrm{GHz}$, scaled to $1.4 \mathrm{GHz}$ by the $\nu^{-0.8}$ power law (neglecting the small thermal bremsstrahlung component), and multiplied by the effective number density of Milky Way galaxies (eq. [54]), is $\mathcal{L}_{g}=9 \times 10^{18} \mathrm{~W} \mathrm{~Hz}^{-1} \mathrm{Mpc}^{-3}$, about half the Condon et al. (2002) measurement of the mean luminosity density of the galaxies (eq. [112]). That is, the evidence is that the Milky Way gives a reasonably good measure of the synchrotron luminosity density of the galaxies, and hence of the cosmic ray luminosities of galaxies. This is not a very direct check of the assumed universality of the cosmic ray lifetime in the source galaxy, of course.

A similar energy may be present in the magnetic field. If the leakage of cosmic rays approximated a fluid flow, magnetic field would leak into intergalactic space with the cosmic rays. Application of equipartition to equation (126) would suggest that the cosmic rms magnetic field strength is

$$
B_{\text {IGM }} \sim 3 \times 10^{-8} \text { Gauß }
$$

If the estimate of the local ratio of the cosmic ray to magnetic field energy density, 8:1 (the magnetic field corresponding to $3 \mu \mathrm{Gauß}$ ), applied to the intergalactic medium, it would lower equation (127) by a factor of three.

The product of the integrated production of supernovae with the characteristic kinetic energy, $E_{\mathrm{KE}} \simeq 1.6 \times 10^{51} \mathrm{erg}$, released in a supernova (Arnett 1996), is an estimate of the integrated kinetic energy production per comoving volume,

$$
\Omega_{\mathrm{SN} \mathrm{KE}} \simeq 10^{-7.3} \text {. }
$$

If the fraction $\epsilon_{\mathrm{cr}}$ of this energy were placed in relativistic intergalactic particles, the present energy density in this component, taking account of the redshift factor, would be

$$
\Omega_{\mathrm{SN} \text { cr }} \simeq 10^{-7.6} \epsilon_{\mathrm{cr}} .
$$

This is consistent with the picture that a fraction $\epsilon_{\mathrm{cr}} \sim 0.2$ of the kinetic energy liberated by supernovae has been deposited in cosmic rays (eq. [126]), which eventually become intergalactic.
Cosmic rays might gain energy from shocks produced by streaming motions of magnetized warm plasma in the vicinity of galaxies (Loeb \& Waxman 2000). However, since the energy available in the plasma (eq. [130] below) is less than the energy liberated by supernovae (eq. [126]), this process is not likely to substantially affect the energy in cosmic rays.

What is the AGN contribution to the cosmic ray energy density? Though AGNs are not major sources at optical wavelengths, their contribution to the radio background is comparable to that of the larger number of normal galaxies (eq. [112). This is an indication of the importance of AGNs for high energy processes. If the cosmic ray energy production by AGNs were proportional to the production of synchrotron radio emission, the energy of cosmic rays from AGN's (including radio elliptical galaxies) would be comparable to, or even larger than, that from the normal galaxies, and our estimate in eq. (126) would have to be doubled. It is important also that AGNs may accelerate cosmic rays to higher energies than do normal galaxies.

We use equation (126) in the inventory. The lower error flag for the entry in Table 1 is based on the evidence that the Milky Way gives a fair measure of galaxy radio luminosities. The larger upper error flag reflects the possibly significant roles of the intergalactic magnetic field and AGNs.

\subsection{Kinetic Energy in the IGM}

Intergalactic baryons have peculiar velocities that are driven by the gravitational field of the dark matter distribution and by nongravitational interactions. The former is part of the primeval energy in category 4 in the inventory. The latter is expected to be most important near the virialized regions of galaxies, where the streaming motion has been largely transformed by shocks into thermal energy at the temperature $T \sim 2 \times 10^{6} \mathrm{~K}$ associated with the nominal velocity dispersion $\sigma=160 \mathrm{~km} \mathrm{~s}^{-1}$ around $L \sim L_{*}$ galaxies (eq. [10]). This shocked matter may be responsible for the O VI absorption lines in the Local Group (Sembach et al. 2003; Cen et al. 2001). The product of the internal energy per unit mass of plasma at this temperature with the mass fraction in entry $3.1 \mathrm{a}$ is

$$
\Omega_{\mathrm{KE}} \simeq 10^{-8.0} .
$$


If an appreciable fraction of the kinetic energy produced by supernovae (eq. [128]) were deposited as kinetic energy in galactic haloes this energy would be dissipated by hydrodynamic processes rather than the cosmological redshift, but still may make a significant addition to equation (130), heating the IGM relative to dark matter.

Baryonic matter well outside the nominal virialized regions of galaxies and larger systems - at distances greater than about $r_{200}$ from clusters of galaxies and $r_{v} \sim 200 \mathrm{kpc}$ from $L \sim L_{*}$ galaxies - is observed as Ly $\alpha$ absorption systems (Penton, Stocke \& Shull 2004). The primeval peculiar motions of this matter are perturbed by photoionisation that produces kinetic temperatures on the order of $10^{4} \mathrm{~K}$. The product of this kinetic energy per unit mass with the intergalactic mass fraction (entry $3.1 \mathrm{~b}$ ) is about one percent of the kinetic energy in the warm intergalactic component (eq. [130]).

We use equation (130) for category 10. We caution, however, that a comparable amount of kinetic energy may be deposited by supernova winds.

\subsection{Electrostatic Energy}

The effect of the electrostatic interaction on the binding energies of atomic nuclei relative to free protons and electrons is taken into account in category 6 , and the electrostatic contribution to the binding energies of white dwarfs is (in principle) part of category 5. The molecular binding energy in objects ranging from dust to asteroids that are held together by the electrostatic interaction deserves separate mention.

The molecular binding energy relative to free atoms in condensed matter is roughly $1 \mathrm{eV}$ per atom. The product of this mass fraction, $\sim 10^{-10}$, with entry 3.12 is

$$
\Omega_{\mathrm{BE}, \text { molecular }} \sim-10^{-16} .
$$

This is the binding energy of condensed matter physics outside strongly self-gravitating systems. We refrain from entering it in Table 1 because the estimate is so small and uncertain, but we offer for comparison the binding energy of the electrons in atoms.

The electrostatic binding energy of the electrons in an $\mathrm{O}$ VI atom is $1.6 \mathrm{keV}$, and the ad- dition of the other five electrons in a neutral oxygen atom increases the binding energy by only 27 percent. That is, it is a reasonable approximation to ignore the states of ionization of the heavy elements and the electrostatic binding energy of neutral hydrogen and helium. The sum over the heavy element masses in entries $1,2,4,5$, and 6 in Table 3, weighted by the neutral atomic binding energies of 15 cosmically conspicuous elements, is

$$
\Omega_{\mathrm{BE}, \text { atoms }}=-10^{-9.7} \text {. }
$$

This is some six orders of magnitude larger than the molecular binding energy in equation (131), and comparable in value to the smallest entries in Table 1.

\section{Discussion}

The convergence of the cosmological tests from redundant constraints on the global cosmological parameters offers a good case for the $\Lambda \mathrm{CDM}$ Friedmann-Lemaître cosmology as a useful approximation to the real world. This is a necessary condition that our compilation of the energy inventory is a meaningful exercise.

We adopt the working assumption that the galaxies are useful tracers of mass, in the sense that inventory entries derived under this assumption are good approximations to reality. This assumption has been widely questioned. However, the recent evidence from weak lensing (eqs. [3] to [7]), with the older evidence from the galaxy relative velocity dispersion at separations $\sim 100 \mathrm{kpc}$ to $1 \mathrm{Mpc}$, and the consistent picture for the virialized parts of field galaxies (eq. [10]), offer what seems to be a reliable case for the use of galaxies as mass tracers. The approach certainly is not exact, and working out more accurate measures of the mass distribution on the scale of galaxies remains an important and fascinating challenge.

Our other conventions for the cosmology are less controversial but may have to be adjusted. For simplicity, we have adopted a fixed distance scale (eq. [1]). We have not presented the scaling of inventory entries with the distance scale, which can be somewhat complicated; this issue is best revisited when the uncertainty in the distance scale is better understood. Our adopted value and formal uncertainty for the matter density parameter, $\Omega_{m}$ (eq. [8]), is in the generally accepted range, but 
there is reason to suspect that it is somewhat overestimated (\$2.1). Again, this is a issue to revisit when the constraints have improved. Our value for the baryon mass density agrees with what is derived from the CMBR anisotropy and the measurements of the primeval deuterium abundance, but it is larger than what is indicated by the primeval helium abundance. We have suggested in $\S 2.2 .2$ that the problem may be with the technical difficulties of the helium measurements, but we will be following the advances in this subject with interest. It would not be surprising to see the discovery of richer physics in the dark sector, and with it an increase in the variety of entries in category 1 , and possibly also some adjustment of other parts of the inventory that depend on the dark sector physics. One should also bear in mind that the alternative pictures of structure formation that were under discussion a decade ago, such as cosmic strings, could be operating as subdominant perturbations to the $\Lambda \mathrm{CDM}$ model. Further progress in testing the model for structure formation will inform our ideas on whether the entries that depend on the theory of structure formation are likely to require adjustment. Each of these issues may be pointing to revisions to the inventory outside our stated errors. On the other hand, the successful network of tests of the cosmology and the model for structure formation leads us to expect that the general framework presented in Table 1 is not likely to change.

We have argued that $6 \pm 1 \%$ of the baryons are in stars and stellar remnants (eqs. [17] and [27] with $h=0.7)$. This small fraction is analyzed in considerable detail in the inventory, in entries that are supported by a network of tests. The estimate in $\S 2.7 .1$ of the mass density in stars uses the optical luminosity density and the stellar-mass-tolight ratio. The luminosity density is checked by measurements of the optical to near infrared intergalactic radiation energy density, and by the measured galaxy counts (§2.7.1). The comparison is not tight, but it shows that the radiation energy density likely is known to \pm 0.3 dex. The conversion from the luminosity density to the stellar mass density depends on the model for the stellar initial mass function. The model we use is checked by the reasonable agreement with the ratio of the white dwarf to subsolar main sequence star densities at the low mass end, and with the global Type II su- pernova rate at the high mass end (2.3.1, 2.3.2). The model for the star formation history is based on the time history of the $\mathrm{H} \alpha$ luminosity density, which is broadly consistent with other measures of the evolution of the star formation rate density (subject to the considerable uncertainty in extinction in the UV). The model is checked by consistency with the accumulated mass in stars (after correction for mass loss; eq. [34]). Yet another check involves the accumulated mass density in heavy elements. The release of nuclear binding energy in the heavy elements, corrected for the loss of radiation energy by the cosmological redshift (eq. [35]), is in satisfactory agreement with the present radiation energy density at near optical and far infrared wavelengths (§2.7.3). This test would fail if there were a substantial amount of radiation energy at wavelengths $1 \mu<\lambda<100 \mu$; improved measurements will be of considerable interest. This test also depends on the stellar production of helium, which is discussed in $\S 2.2 .2$, in connection with the constraint on the baryon mass density from the primeval helium abundance, and in $§ 2.8 .1$, from an analysis of the products of stellar evolution. The results seem reasonably consistent. The network of checks is complicated, but that is what lends credence to the results.

It is well to pause to consider Arp's (1965) cautionary remark, that there may be extragalactic objects with sizes too small to be readily distinguished from stars - an example is the quasistellar objects Arp mentions in a note added in proof - or with surface brightnesses too low to be readily seen against the foreground - an example is the intracluster light in the Virgo cluster (Arp \& Bertola 1971). The quasar remnants could have contained a significant baryon mass, if the mass conversion efficiency $\epsilon_{n}$ had been close to unity (eq. [73]), but we now know that that is difficult to reconcile with the integrated quasar energy emission (eq. [83]). There are low surface brightness objects (e.g McGaugh, Schombert, \& Bothun 1995), but the surface brightness of the extragalactic sky shows that they cannot largely affect our estimate of the mean luminosity density (§2.7.1). Substellar objects might be counted as part of Arp's cautionary remark, but they are detectable in nearby galaxies by weak gravitational lensing (or MACHOs, as discussed by Alcock et al. 2000 and references therein). That is, a half 
century ago it was not clear that it is feasible to establish a fair observational census of the stars. Now it appears that the observational conditions allow it: the closure of our inventory suggests that missing or unknown components cannot be energetically very significant.

The baryon number density — excluding baryons that may have been sequestered prior to light element production — seems to be reliably constrained, but the states of most of the baryons are not yet observationally well documented. The picture that galaxies trace mass leads us to expect that about half the dark matter is gathered near and within the virialized parts of the galaxies (eq. [13]). Entries 3.1 and 3.2 in Table 1 are based on the assumption that the baryons are similarly placed, in the diffuse states observed as hot plasma in clusters of galaxies and in warm and hot plasma in and around groups of galaxies (eq. [56]). This has not yet been convincingly observationally demonstrated. We have not made much use of the predicted states of the baryons from numerical simulations, because we do not know how to judge the reliability of the predictions on relatively small scales. As the computations and their observational checks improve this will become clearer, and the results may be expected to improve this part of the inventory.

The advances in observations of the massive compact objects in the centers of galaxies allow an improved test of the idea that these objects are the black hole remnants of quasars and AGNs that are powered by gravitational accretion. Our estimates of the mass density in black holes and the luminosity density in quasars require that the quasar mass conversion efficiency is $\epsilon_{n} \sim 0.02$ (eq. [86]), close to the efficiency of production of gravitational radiation (§2.7.6). If the estimate of the quasar luminosity density has missed a significant population of optically faint AGNs then our value of $\epsilon_{n}$ is biased low. The quasar luminosity density is quite uncertain, as is the actual value of $\epsilon_{n}$, but it is encouraging that the present result is as close as it is to conventional ideas about the quasar emission mechanism.

The efficiency of conversion of mass into electromagnetic radiation in the formation of stellar mass black holes is constrained to be less than a few percent by the observed energy density in electromagnetic radiation and neutrinos. The in- terpretation of this constraint awaits development of the theory of the astrophysical processes of formation of stellar mass black holes. Our estimate of the gravitational binding energy released by core collapse supernovae (entry 8.3) is comparable to the energy released in the formation of the massive black hole quasar remnants, and it is not very much less than the upper bound from neutrino detectors. Progress here will be followed with interest.

The largest energy densities in the inventory are in dark matter and dark energy. Any empirical hints to the natures of these components will be followed with great interest. One possibility is that the observations of microlensing toward the Magellanic Clouds have detected massive compact dark objects (Alcock et al. 2000; Afonso et al. 2003). We have adopted the conservative interpretation, that the lenses are baryonic - stars and stellar remnants — with standard mass functions, and that the high lensing rate is an accident of the distributions of source and lensing stars. A demonstration of the apparently simpler interpretation, that the lenses contribute some $20 \%$ of the mass of the halo of the Milky Way, and are baryonic, perhaps white dwarfs, would considerably upset our considerations of the stellar baryon budget; a demonstration that the lenses are nonbaryonic would profoundly affect thinking about dark matter and the early universe.

Though there are large gaps in our understanding of the cosmic energy inventory, most notably in the dark sector, there also is an impressively broad observational basis for many parts of the inventory. Ten years ago the basis was considerably smaller, and we can be sure that ten years from now the observations will allow more entries and a richer network of crosschecks of the interpretations. Progress will be uneven, of course, as one sees by considering the prospects for further checks of the neutrino energy inventory. With the recent demonstration that the solar neutrino luminosity agrees with standard physics augmented by neutrino masses and mixing angles (Bahcall 2003 and references herein) we may now be confident that the theory of neutrino production by stellar nuclear burning is reliably tested even in the absence of a direct observation of stellar neutrinos from remote stars. The neutrinos produced by plasma processes in white dwarf formation are, 
while an enormous energy density, too soft for detection by any conceivable method: estimates of this component of the inventory will have to continue to rely on the theory. Neutrinos released in stellar core collapse have been detected (Hirata et al. 1987; Bionta et al. 1987), and the integrated background of relic neutrinos from this process is expected to be within experimental reach. The detection would complete another valuable check of the cosmic energy transactions.

Parts of our inventory can be improved on the basis of what is available within existing computations, or could be readily developed from existing work, but that we could not readily assemble. In particular, the theoretical analyses of stellar remnants and the other products of stellar evolution ( $\S \S 2.3 .1$ and 2.8), with special attention to the white dwarfs that store so much of the heavy elements, will have to be done better. Another example is the heavy element abundances, which can be classified by element type, as oxygen which has a specific spectroscopic importance and the $\alpha$-elements and the iron group, and by environment, as white dwarfs, main sequence stars, and interstellar and intergalactic space.

It is also worth stating explicitly that we may expect that, as in the past, there will be qualitatively new experimental or observational developments - perhaps the detection of gravitational radiation, or the identification of dark matter particles - that substantially affect the directions of research and the development of the cosmic energy inventory.

\section{Concluding Remarks}

A half century ago we had a largely conjectural picture of the large-scale structure of the physical universe, of its material contents, and of the main process that drive transformations among the states of matter and radiation. Now our world picture has a substantial basis in experiments and observations and the attendant theories. This has been an evolutionary process: none the entries in the inventory in Table 1 requires a substantial departure from ideas that are under discussion, and in many cases have been in the literature for decades. The important new development is that we now have observational support for the many entries in Table 1, and a network of tests that demonstrate that a considerable part of the inventory is a believable approximation. Continued advances in the observational and theoretical basis for the inventory surely will yield unexpected revisions and additions; we are attempting to draw large conclusions from limited observations of an exceedingly complex universe. However, the big surprise at the moment is that it is now possible to find an inventory with observational support for the largest $\sim 40$ forms of energy.

We have benefited by advice from Charles Alcock, Dave Arnett, John Bahcall, Michael Blanton, Pierre Bergeron, Bruce Draine, Doug Finkbeiner, Peter Goldreich, Andy Gould, Vicky Kalogera, Kim Griest, Craig Hogan, David Hogg, Guinevere Kauffmann, Julian Krolik, Jim Liebert, Geoff Marcy, Bruce Partridge, Martin Rees, Neill Reid, Aldo Serenelli, Masaru Shibata, Mike Shull, Gary Steigman, Paul Steinhardt, Stuart Shapiro, Tomonori Totani, Scott Tremaine, Simon White, and the referee.

PJEP thanks the Japan Society for Promotion of Science for supporting his visit to Japan, where this work was initiated. MF received support for this work from the Monell Foundation at the Princeton Institute for Advanced Study, and a Grant in Aid from the Japanese Ministry of Education at the University of Tokyo. 


\section{A. A Few Astrophysical Quantities for the Milky Way}

The current best value for the distance to the Galactic center is

$$
R_{0}=7.94 \pm 0.42 \mathrm{kpc},
$$

from measurements of the orbit - proper motion and redshift — of a star close to Sgr A* (Eisenhauer et al. 2003). The product of this distance with the measurement of the proper motion of Sgr A* by Reid \& Brunthaler (2004) is $241 \mathrm{~km} \mathrm{~s}^{-1}$. This velocity corrected for the solar proper motion is our adopted value of the circular speed of the Milky Way at the Solar circle,

$$
\Theta_{0}=234 \pm 13 \mathrm{~km} \mathrm{~s}^{-1} .
$$

Traditionally, one invokes the Oort model to infer the circular speed, but Olling \& Dehnen (2003) find that the current best result depends substantially on the stellar populations used in the analysis.

The circular velocity yields an estimate of the optical luminosity of the Milky Way via the Tully-Fisher relation. To convert the optically measured maximum rotation velocity $V_{\text {rot }}$ to the $20 \%$ line width of HI measurements $W_{20}$, we use the relation

$$
W_{20} / 2=(0.93 \pm 0.02) V_{\text {rot }}+(27 \pm 1.5) \mathrm{km} \mathrm{s}^{-1} .
$$

derived from the data given by Mathewson \& Ford (1996). From the Galactic rotation curve from HI and CO terminal velocities (using Fig. 9.17 of Binney \& Merrifield 1998), we estimate that the maximum rotation velocity of the Milky Way is $V_{\max }=1.03 \Theta_{0}=241 \pm 13 \mathrm{~km} \mathrm{~s}^{-1}$. Identifying $V_{\max }=V_{\text {rot }}$, we find

$$
W_{20}(\mathrm{MW})=502 \pm 30 \mathrm{~km} \mathrm{~s}^{-1} \text {. }
$$

From the COBE-DIRBE integrated light for the Milky Way in the near infrared $(\mathrm{J}, \mathrm{K}, \mathrm{L})$ wavelength bands, Malhotra et al. (1996) showed that the Milky Way is on the standard Tully-Fisher relation in these wave length bands, slightly on the brighter side but within the dispersion around the relation. Assuming that the Milky Way has the standard color of spiral galaxies, we may expect that the luminosity in the B band also satisfies the Tully-Fisher relation for the B band. Using the Sakai et al. (2000) local calibration,

$$
M_{B_{T}^{c}}=-19.80-7.97\left(\log W_{20}-2.5\right),
$$

we find

$$
L_{B^{c}}(\mathrm{MW})=10^{10.74 \pm 0.19} L_{\odot},
$$

where we use $B_{\odot}=5.46$, and the error is dominated by the intrinsic dispersion ( $\left.0.43 \mathrm{mag}\right)$ of the B-band Tully-Fisher relation. The superscript $c$ stands for extinction-free quantities.

An alternative estimate of the B-band luminosity uses the colour transformation applied to the DIRBE integrated K-band luminosity of the Milky Way. A comparison of the samples of Malhotra et al.(1996) and Sakai et al. (2000) indicates $B_{T}^{c}-K^{c}=2.84 \pm 0.10$ for typical Sbc galaxies. Thus $K^{c}(\mathrm{MW})=-23.95 \pm 0.25$ yields $B_{\mathrm{T}}^{c}=-21.1 \pm 0.3$, or

$$
L_{B^{c}}(\mathrm{MW})=10^{10.63 \pm 0.13} L_{\odot} .
$$

Combining the two, our recommended value is

$$
L_{B^{c}}(\mathrm{MW})=4.6 \pm 1.4 \times 10^{10} L_{\odot} .
$$

A value often quoted in the literature, $L_{B}^{c}=2.3 \pm 0.6 \times 10^{10} L_{\odot}$ (van den Bergh 1988), is 1.5 standard deviations below equation (A8).

The luminosity obtained here is the extinction-free value. When it is compared with $L^{*}$ that appears in the luminosity function, we must take account of internal extinction. For consistency we use the average 
value of the internal extinction correction in the Sakai et al. (2000) local calibration, $A_{B}^{\text {int }}=0.44$ mag, or an attenuation factor 1.5. This means that $L_{B}(\mathrm{MW})=1.40 \mathrm{~L}_{\mathrm{B}}^{*}$ at $h=0.7$, and that the effective number density of Milky Way galaxies is the value in equation $(54), \mathcal{L}_{\mathcal{B}} / L_{B}(\mathrm{MW})=0.013 \mathrm{~h}^{3} \mathrm{Mpc}^{-3}$. 


\section{REFERENCES}

Abazajian, K., et al. 2003, AJ, 126, 2081

Afonso, C. et al. 2003, A\&A, 400, 951

Aharonian, F. A., et al. 1999, A\&A, 349, 11

Alcock, C. et al. (The MACHO Collaboration) 2000, ApJ, 542, 281

Allende Prieto, C., Lambert, D. L., \& Asplund, M. 2001, ApJ, 556, L63

Arnett, D. 1996, Supernovae and Nucleosynthesis: An Investigation of the History of Matter, from the Big Bang to the Present (Princeton: Princeton University Press)

Arp, H. 1965, ApJ, 142, 402

Arp, H. \& Bertola, F. 1971, ApJ, 163, 195

Bahcall, J. N. 2003, astro-ph/0310030

Bahcall, J. N. \& Peña-Garay, C. 2003, JHEP 0311, 004

Bahcall, J. N. \& Pinsonneault, M. H. 2004, Phys. Rev. Lett. 92, 12130

Bahcall, J. N., Pinsonneault, M. H., \& Basu, S. 2001, ApJ, 555, 990 (BP2000)

Bahcall, N. A. \& Bode, P. 2003, ApJ, 588, L1

Bahcall, N. A. \& Cen, R. 1993, ApJ, 407, L49

Bahcall, N. A., Lubin, L. M., \& Dorman, V. 1995, ApJ, 447, L8

Bahcall, N. A., et al. 2003, ApJ, 585, 182

Baker, J., Brügmann, B., Campanelli, M. Lousto, C. O. \& Takahashi, R. 2001, Phys. Rev. Lett. 87,121103

Basu, B. \& Lynden-Bell, D. 1990, QJRAS, 31, 359

Bennett, C. L. et al. 2003a, ApJS, 148, 1

Bennett, C. L., et al. 2003b, ApJS, 148, 97

Bernstein, R. A., Freedman, W. L., \& Madore, B. F. 2002, ApJ, 571, 56

Beuermann, K., Kanbach, G., \& Berkhuijsen, E. M. 1985, A\&A, 153, 17
Binney , J. \& Merrifield, M. 1998, Galactic Astronomy (Princeton: Princeton University Press)

Bionta, R. M., Blewitt, G., Bratton, C. B., Caspere, D., \& Ciocio, A. 1987, Physical Review Letters, 58, 1494

Blanton, M. R., et al. 2003, ApJ, 592, 819

Bosma, A. 1981, AJ, 86, 1825

Branch, D. 1992, ApJ, 392, 35

Bridle, A. H. 1967, MNRAS, 136, 219

Bruzual, G. \& Charlot, S. 2003, MNRAS, 344, 1000

Burgasser, A. J., Kirkpatrick, J. D., McElwain, M. W., Cutri, R. M., \& Skrutskie, M. F. 2003, AJ, 125,850

Burgasser, A. J., McElwain, M. W., Kirkpatrick, J. D., Cruz, K. L., Tinney, C. G., \& Reid, I. N. 2004, AJ, 127, 2856

Burrows, A. 1990, in Supernovae, ed. by Petschek, A. G. (Berlin: Springer), 143

Burrows, A., Hubbard, W. B., Lunine, J. I., \& Liebert, J. 2001, Reviews of Modern Physics, 73,719

Calura, F. \& Matteucci, F. 2004, MNRAS, 350, 351

Cappellaro, E., Evans, R., \& Turatto, M. 1999, A\&A, 351, 459

Cappellaro, E., Turatto, M., Tsvetkov, D. Y., Bartunov, O. S., Pollas, C., Evans, R., \& Hamuy, M. 1997, A\&A, 322, 431

Cen, R., Tripp, T. M., Ostriker, J. P., \& Jenkins, E. B. 2001, ApJ, 559, L5

Chabrier, G. 2003, PASP, 115, 763

Chen, H., Lanzetta, K. M., Webb, J. K., \& Barcons, X. 2001, ApJ, 559, 654

Chiosi, C., Bertelli, G., \& Bressan, A. 1992, ARA\&A, 30, 235

Chokshi, A. \& Turner, E. L. 1992, MNRAS, 259, 421 
Churchill, C. W., Vogt, S. S., \& Charlton, J. C. 2003, AJ, 125, 98

Claver, C. F., Liebert, J., Bergeron, P., \& Koester, D. 2001, ApJ, 563, 987

Cole, S. et al. 2001, MNRAS, 326, 255

Colless, M., et al. 2001, MNRAS, 328, 1039

Condon, J. J. 1992, ARA\&A, 30, 575

Condon, J. J., Cotton, W. D., \& Broderick, J. J. 2002, AJ, 124, 675

Condon, J. J., Cotton, W. D., Greisen, E. W., Yin, Q. F., Perley, R. A., Taylor, G. B., \& Broderick, J. J. 1998, AJ, 115, 1693

Cox, A. N. (ed) 2000, Allen's Astrophysical Quantities, 4th ed. (Berlin: Springer)

Cox, J. P. \& Giulli, R. T. 1968, Principles of Stellar Structure, Vol. 2 (New York: Gordon and Breach)

Croom, S. M., Smith, R. J., Boyle, B. J., Shanks, T., Miller, L \& Outram 2004, astro-ph/0403040

Davis, M. \& Peebles, P. J. E. 1983, ApJ, 267, 465

De Luca, A. \& Molendi, S. 2004, A\&A, 419, 837

de Sitter, W. 1917, MNRAS, 78, 3

de Vaucouleurs, G. \& Olson, D. W. 1982, ApJ, 256,346

Draine, B. T. 2003, ARA\&A, 41, 241

Eguchi, K. et al. 2003, Phys. Rev. Lett. 90, 021802

Einstein, A. 1917, Sitzungsber Preuß Akad Wiss, p. 142 [English translation in The Principle of Relativity (Dover, New York, 1952), p. 177]

Eisenhauer, F., Schödel, R., Genzel, R., Ott, T., Tecza, M., Abuter, R., Eckart, A., \& Alexander, T. 2003, ApJ, 597, L121

Elvis, M., et al. 1994, ApJS, 95, 1

Ezer, D. \& Cameron, A. G. W. 1967, Canadian Journal of Physics, 45, 3429

Faber, J. A., Grandclément, P., Rasio, F. A., \& Taniguchi, K. 2002, Physical Review Letters, 89, 231102
Fan, X., et al. 2001, AJ, 121, 54

Fang, T., Marshall, H. L., Lee, J. C., Davis, D. S. \& Canizares, C. R. 2002, ApJ, 572, L127

Fang, T., Sembach, K. R., \& Canizares, C. R. 2003, ApJ, 586, L49

Ferrarese, L. 2002, Proceedings, Second KIAS Astrophysics Workshop, Seoul, 2001, September 3-7, Singapore: World Scientific, edited by Lee, C.-H., 3

Finkbeiner, D. P., Davis, M., \& Schlegel, D. J. 2000, ApJ, 544, 81

Fixsen, D. J., Cheng, E. S., Gales, J. M., Mather, J. C., Shafer, R. A., \& Wright, E. L. 1996, ApJ, 473,576

Fixsen, D. J., Dwek, E., Mather, J. C., Bennett, C. L., \& Shafer, R. A. 1998, ApJ, 508, 123

Fomalont, E. B., Kellermann, K. I., Partridge, R. B., Windhorst, R. A., \& Richards, E. A. 2002, AJ, 123, 2402

Freedman, W. L., et al. 2001, ApJ, 553, 47

Fukazawa, Y., Makishima, K., Tamura, T., Ezawa, H., Xu, H., Ikebe, Y., Kikuchi, K., \& Ohashi, T. 1998, PASJ, 50, 187

Fukuda, Y., et al. 1998, Physical Review Letters, 81,1562

Fukugita, M. 2001, NATO ASIC Proc. 565: Structure Formation in the Universe, 93

Fukugita, M., Hogan, C. J., \& Peebles, P. J. E. 1998, ApJ, 503, 518 (FHP)

Fukugita, M. \& Kawasaki, M. 2003, MNRAS, 340, L7

Gallego, J., Zamorano, J., Aragon-Salamanca, A., \& Rego, M. 1995, ApJ, 455, L1

Gal-Yam, A. \& Maoz, D. 2004, MNRAS, 347, 942

Gao, L., Loeb, A., Peebles, P. J. E., White, S. D. M. \& Jenkins, A. 2003, astro-ph/0312499

Garcia-Munoz, M., Mason, G. M., \& Simpson, J. A. 1977, ApJ, 217, 859 
Ginzburg, V. I. \& Syrovatskii, S. I. 1964, The Origin of Cosmic Rays (Oxford: Pergamon)

Glazebrook, K., Blake, C., Economou, F., Lilly, S., \& Colless, M. 1999, MNRAS, 306, 843

Glazebrook, K., et al. 2003, ApJ, 587, 55

Gott, J. R., Schramm, D. N., Tinsley, B. M., \& Gunn, J. E. 1974, ApJ, 194, 543

Gebhardt, K., et al. 2000, ApJ, 539, L13

Gould, A., Bahcall, J. N., \& Flynn, C. 1996, ApJ, 465,759

Grevesse, N. \& Sauval, A. J. 2000, Origin of Elements in the Solar System, Implications of Post1957 Observations, Proceedings of the International Symposium. ed. O. Manuel. (Dordrecht: Kluwer Academic Publishers), 261

Gruber, D. E., Matteson, J. L., Peterson, L. E., \& Jung, G. V. 1999, ApJ, 520, 124

Gush, H. P., Halpern, M., \& Wishnow, E. H. 1990, Physical Review Letters, 65, 537

Haarsma, D. B. \& Partridge, R. B. 1998, ApJ, 503, L5

Haarsma, D. B., Partridge, R. B., Windhorst, R. A., \& Richards, E. A. 2000, ApJ, 544, 641

Harris, H. C., et al. 2003, AJ, 126, 1023

Haslam, C. G. T., Stoffel, H., Salter, C. J., \& Wilson, W. E. 1982, A\&AS, 47, 1

Hauser, M. G. \& Dwek, E. 2001, ARA\&A, 39, 249

Hauser, M. G., et al. 1998, ApJ, 508, 25

Hawkins, E. et al. 2003, MNRAS 346, 78

Hayashi, C., \& Nakano, T. 1963, Prog. Theor. Phys. 30, 460

Heavens, A., Panter, B., Jimenez, R., \& Dunlop, J. 2004, Nature, 428, 625

Heger, A., Woosley, S. E., Fryer, C. L., \& Langer, N. 2003, From Twilight to Highlight: The Physics of Supernovae, 3 (astro-ph/0211062)

Henry, R. C. 1999, ApJ, 516, L49
Hils, D., Bender, P. L., \& Webbink, R. F. 1990, ApJ, 360, 75

Hirata, K., Kajita, T., Koshiba, M., Nakahata, M., \& Oyama, Y. 1987, Physical Review Letters, 58, 1490

Hubble, E. P. 1929, Proc Nat Acad Sci, 15, 168

Hubble, E. P. 1934, ApJ, 79, 8

Hubble, E. P. 1936, The Realm of the Nebulae (New Haven: Yale University Press)

Huchra, J., Jarrett, T., Skrustkie, M., Cutri, R., Schneider, S., Macri, L., Steining, R., \& Mader, J. 2003, IAU Symposium, 216, 174

Hughes, S. A., Marka, S., Bender, P. L. \& Hogan, C. J. 2001, in Proceedings of Snowmass 2001, APS/DPF/DPB Summer Study on the Future of Particle Physics, eConf C010630: p402 (also astro-ph/0110349)

Irvine, W. M. 1961, Doctoral Dissertation, Harvard University

Ivezić, Ž., et al. 2000, AJ, 120, 963

Izotov, Y. I. \& Thuan, T. X. 2004, ApJ, 602, 200

Jaffe, A. H. \& Backer, D. C. 2003, ApJ, 583, 616

Janka, H.-T. \& Hillebrandt, W. 1989, A\&A, 224, 49

Jimenez, R., Flynn, C., MacDonald, J., \& Gibson, B. K. 2003, Science, 299, 1552

Kalogera, V., et al. 2004a, ApJ, 601, L179

Kalogera, V., et al. 2004b, astro-ph/0312101, v. 3

Kameda, J. et al. 2001, in Proc. International Cosmic Ray Conference, Hamburg 2001, ed. K.-H. Kampert, G. Heizelmann \& C. Spiering (Katlenburg-Lindau: Copernicus Gesellschaft, 2001) Vol. 3, 1057

Kaspi, V. M., Taylor, J. H., \& Ryba, M. F. 1994, ApJ, 428, 713

Kauffmann, G. et al. 2003, MNRAS, 341, 33

Kennicutt, R. C. 1983, ApJ, 272, 54

Keres, D., Yun, M. S., \& Young, J. S. 2003, ApJ, 582, 659 
Kippenhahn, R., Thomas, H. C., \& Weigert, A. 1965, Zeits. für Astrophysik, 61, 241

Kirkman, D., Tytler, D., Suzuki, N., O’Meara, J. M., \& Lubin, D. 2003, ApJS, 149, 1

Kneiske, T. M., Mannheim, K., \& Hartmann, D. H. 2002, A\&A, 386, 1

Kobulnicky, H. A. \& Zaritsky, D. 1999, ApJ, 511, 118

Kochanek, C. S. et al. 2001, ApJ, 560, 566

Konopelko, A., Mastichiadis, A., Kirk, J., de Jager, O. C., \& Stecker, F. W. 2003, ApJ, 597, 851

Kormendy, J. \& Richstone, D. 1995, ARA\&A, 33, 581

Kroupa, P. 2001, MNRAS, 322, 231

Kumar, S. S. 1963, ApJ, 137, 1121

LaRoque, S. J., et al. 2003, ApJ, 583, 559

Layzer, D. 1963, ApJ, 138, 174

Lilly, S. J., Le Fevre, O., Hammer, F., \& Crampton, D. 1996, ApJ, 460, L1

Loeb, A. \& Waxman, E. 2000, Nature, 405, 156

Lommen, A. N. \& Backer, D. C. 2001, BAAS, 33, 1347

Longair, M. 1995, Proceedings of the Extragalactic Background Radiation meeting, Baltimore, 1993 May 18-20, Space Telescope Science Institute Symposium Series, meeting, ed. Calzetti, D., Livio, M., \& Madau, P. (Cambridge: Cambridge University Press), 223

Machalski, J. \& Godlowski, W. 2000, A\&A, 360, 463

Madau, P., della Valle, M., \& Panagia, N. 1998, MNRAS, 297, L17

Madau, P., Ferguson, H. C., Dickinson, M. E., Giavalisco, M., Steidel, C. C., \& Fruchter, A. 1996, MNRAS, 283, 1388

Madau, P. \& Pozzetti, L. 2000, MNRAS, 312, L9

Malhotra, S., Spergel, D. N., Rhoads, J. E., \& Li, J. 1996, ApJ, 473, 687
Marcy, G. W. \& Butler, R. P. 2000, PASP, 112, 137

Mather, J. C., et al. 1990, ApJ, 354, L37

Mather, J. C., Fixsen, D. J., Shafer, R. A., Mosier, C., \& Wilkinson, D. T. 1999, ApJ, 512, 511

Mathewson, D. S. \& Ford, V. L. 1996, ApJS, 107, 97

Mattila, K. 1990, IAU Symp. 139: The Galactic and Extragalactic Background Radiation, 139, 257

McGaugh, S. S., Schombert, J. M., \& Bothun, G. D. 1995, AJ, 109, 2019

McKay, T. A. et al. 2001, astro-ph/0108013

Merritt, D. \& Ferrarese, L. 2001, ApJ, 547, 140

Molster, F. J., Waters, L. B. F. M., Tielens, A. G. G. M., Koike, C., \& Chihara, H. 2002, A\&A, 382, 241

Mushotzky, R. F., Cowie, L. L., Barger, A. J., \& Arnaud, K. A. 2000, Nature, 404, 459

Mushotzky, R. F. \& Loewenstein, M. 1997, ApJ, 481, L63

Nakamura, O., Fukugita, M., Brinkmann, J., \& Schneider, D. P. 2004, AJ, 127, 2511

Nakamura, O., Fukugita, M., Yasuda, N., Loveday, J., Brinkmann, J., Schneider, D. P., Shimasaku, K., \& SubbaRao, M. 2003, AJ, 125, 1682

Nice, D. J., Splaver, E. M., \& Stairs, I. H. 2003, ASP Conf. Ser. 302: Radio Pulsars, 75

Nomoto, K. 1984, ApJ, 277, 791

Olling, R. P. \& Dehnen, W. 2003, ApJ, 599, 275

Pagel, B. E. J. \& Portinari, L. 1998, MNRAS, 298, 747

Pain, R., et al. 2002, ApJ, 577, 120

Partridge, R. B. \& Peebles, P. J. E. 1967, ApJ, 148,377 
Peacock, J. A. 1995, Proceedings of the Extragalactic Background Radiation meeting, Baltimore, 1993 May 18-20, Space Telescope Science Institute Symposium Series, meeting, ed. by Calzetti, D., Livio, M., \& Madau, P. (Cambridge: Cambridge University Press), 237

Peebles, P. J. E. 1986, Nature, 321, 27

Peebles, P. J. E. \& Partridge, R. B. 1967, ApJ, 148,713

Peebles, P. J. \& Ratra, B. 2003, Reviews of Modern Physics, 75, 559

Peimbert, M., Peimbert, A., \& Ruiz, M. T. 2000, ApJ, 541, 688

Penton, S. V., Stocke, J. T. \& Shull, J. M. 2004, astro-ph/0401036

Quinn, J., et al. 1996, ApJ, 456, L83

Reid, I. N., Gizis, J. E., \& Hawley, S. L. 2002, AJ, 124, 2721

Reid, M. J. \& Brunthaler, A. 2004, astro$\mathrm{ph} / 0408107$

Reimers, D. \& Koester, D. 1982, A\&A, 116, 341

Reiprich, T. H. \& Böhringer, H. 2002, ApJ, 567, 716

Richter, P., Savage, B. D., Sembach, K. R., Tripp, T. M., \& Jenkins, E. B. 2003, ASSL Vol. 281: The IGM/Galaxy Connection. The Distribution of Baryons at $\mathrm{z}=0,85$

Riess, A. et al. 2004, ApJ, 607, 665

Rudnick, G., et al. 2003, ApJ, 599, 847

Sadler, E. M., et al. 2002, MNRAS, 329, 227

Sakai, S., et al. 2000, ApJ, 529, 698

Salpeter, E. E. 1955, ApJ, 121, 161

Salucci, P. \& Persic, M. 1999, MNRAS, 309, 923

Salucci, P., Szuszkiewicz, E., Monaco, P., \& Danese, L. 1999, MNRAS, 307, 637

Scalo, J. M. 1986, Fundamentals of Cosmic Physics, 11, 1
Schwarzschild, M. 1958, Structure and Evolution of the Stars (Princeton: Princeton University Press)

Searle, L., Sargent, W. L. W., \& Bagnuolo, W. G. 1973, ApJ, 179, 427

Schneider, R., Ferrari, V., Matarrese, S., \& Portegies Zwart, S. F. 2001, MNRAS, 324, 797

Sembach, K. R., et al. 2003, ApJS, 146, 165

Shapiro, S. L. \& Teukolsky, S. A. 1983, Black Holes, White Dwarfs, and Neutron Stars: The Physics of Compact Objects (New York: WileyInterscience)

Sheth, R. K., et al. 2003, ApJ, 594, 225

Shibata, M. \& Uryū, K. 2002, Progress of Theoretical Physics, 107, 265

Shull, J. M. 2003, ASSL Vol. 281: The IGM/Galaxy Connection. The Distribution of Baryons at $\mathrm{z}=0,1$

Shull, J. M., Tumlinson, J., \& Giroux, M. L. 2003, ApJ, 594, L107

Simon, A. J. B. 1978, MNRAS, 184, 537

Sołtan, A. 1982, MNRAS, 200, 115

Spergel, D. N. et al. 2003, ApJS, 148, 175

Steidel, C. C., Adelberger, K. L., Giavalisco, M., Dickinson, M., \& Pettini, M. 1999, ApJ, 519, 1

Steidel, C. C., Dickinson, M., \& Persson, S. E. 1994, ApJ, 437, L75

Tammann, G. A., Löeffler, W., \& Schröeder, A. 1994, ApJS, 92, 487

Tegmark, M. et al. 2004a, Physical Review D69, 103501

Tegmark, M. et al. 2004b, ApJ, 606, 702

Thorsett, S. E. \& Chakrabarty, D. 1999, ApJ, 512, 288

Thorsett, S. E. \& Dewey, R, J. 1996, Phys Rev D53, 3468

Tinsley, B. M. 1973, A\&A, 24, 89

Tremaine, S., et al. 2002, ApJ, 574, 740 
Tripp, T. M., Savage, B. D., \& Jenkins, E. B. 2000, ApJ, 534, L1

Trujillo, C. A., Jewitt, D. C., \& Luu, J. X. 2001, AJ, 122, 457

van den Bergh, S. 1988, Comments on Astrophysics, 12,131

van den Bergh, S. \& McClure, R. D. 1994, ApJ, 425,205

Vennes, S., Smith, R. J., Boyle, B. J., Croom, S. M., Kawka, A., Shanks, T., Miller, L., \& Loaring, N. 2002, MNRAS, 335, 673

Verde, L., et al. 2002, MNRAS, 335, 432

Waldram, E. M., Pooley, G. G., Grainge, K. J. B., Jones, M. E., Saunders, R. D. E., Scott, P. F., \& Taylor, A. C. 2003, MNRAS, 342, 915

Weaver, T. A., Zimmerman, G. B., \& Woosley, S. E. 1978, ApJ, 225, 1021

Webber, W. R. 1998, ApJ, 506, 329

Weidemann, V. 2000, A\&A, 363, 647

Weiler, K. W., Sramek, R. A., Panagia, N., van der Hulst, J. M., \& Salvati, M. 1986, ApJ, 301, 790

Whelan, J. \& Iben, I. J. 1973, ApJ, 186, 1007

White, D. A. 2000, MNRAS, 312, 663

Willott, C. J., Rawlings, S., Blundell, K. M., Lacy, M., \& Eales, S. A. 2001, MNRAS, 322, 536

Worsley, M. A. et al. 2004, astro-ph/0404273

Wright, E. L. \& Reese, E. D. 2000, ApJ, 545, 43

Wyithe, J. S. B. and Loeb, A. 2003, ApJ, 590,691

Yanasak, N. E., et al. 2001, ApJ, 563, 768

York, D. G., et al. 2000, AJ, 120, 1579

Zhang, P., Pen. W.-L. \& Trac, H. 2004, astro$\mathrm{ph} / 0402115$

Zwaan, M. A. et al. 2003, AJ, 125, 2842

Zwicky, F. 1933, Helv. Phys. Acta, 6, 110

This 2-column preprint was prepared with the AAS LATEX macros v 5.2 . 